\title{
Deformation of a Circular Elastic Tube between Two Parallel Bars: Quasi-Analytical Geometrical Ring Models
}

\author{
A. Van Hirtum \\ GIPSA-lab, UMR CNRS 5216, Grenoble University, 38000 Grenoble, France \\ Correspondence should be addressed to A. Van Hirtum; annemie.vanhirtum@gipsa-lab.grenoble-inp.fr
}

Received 8 March 2015; Accepted 21 April 2015

Academic Editor: Francesco Tornabene

Copyright (C) 2015 A. Van Hirtum. This is an open access article distributed under the Creative Commons Attribution License, which permits unrestricted use, distribution, and reproduction in any medium, provided the original work is properly cited.

\begin{abstract}
Several engineering problems are confronted with elastic tubes. In the current work, homothetic quasi-analytical geometrical ring models, ellipse, stadium, and peanut, are formulated allowing a computationally low cost ring shape estimation as a function of a single parameter, that is, the pinching degree. The dynamics of main geometrical parameters due to the model choice is discussed. Next, the ring models are applied to each cross section of a circular elastic tube compressed between two parallel bars for pinching efforts between $40 \%$ and $95 \%$. The characteristic error yields less than $4 \%$ of the tubes diameter when the stadium model was used.
\end{abstract}

\section{Introduction}

An accurate description of a constricted channel's geometry and related geometrical features is often crucial and, therefore, a recurrent problem in different engineering disciplines. Geometrical features will affect main fluid flow characteristics related to inertia, boundary layer development, flow detachment, or jet formation downstream from the constricted channel portion [1]. The same way, the accuracy of wave related problems will depend on the channels or waveguides geometry since it will influence among others the propagation or evanescence of higher order propagation modes [2]. Knowledge of the channel geometry is in particular an obstacle when dealing with natural occurring fluid flow or wave propagation through elastic channels for which no geometrical design is possible and in addition the shape can rapidly vary such as is the case for biological channel flows (lower and upper airway respiratory or blood circulation system) and related biomechanical phenomena.

Partly motivated by the large number of applications, a substantial amount of research is performed (see, e.g., [38]) on elastic tube modeling for a variety of boundary and loading conditions since the pioneering work [9-11] on thinwalled elastic tubes. Despite those efforts and the increased insight in the stability and bifurcation of circular elastic tubes, no analytical model is capable of providing geometrical features for channels subjected to rapidly varying loading resulting in small as well as large deformations of thin as well as thick wall tubes of variable length. Therefore, in the current work quasi-analytical parameterised geometrical ring models are used in order to provide geometrical features of a compressed elastic tube. Although those models do not give insight in the dynamics of the elastic ring, they do allow, due to their simplicity, to avoid or overcome some of the limitations of more advantaged analytical models depending on boundary conditions (loading, geometry, ...). Therefore, when solely the ring shape (or an associated geometrical parameters) is searched, quasi-analytical geometrical models might be of interest in providing an estimation of the shape at a negligible computational cost while the parameterization favors usage in combination with other analytical model approaches exploiting a limited number of input parameters or when an initialization of the ring shape is needed. This might, for example, be the case for real-life applications for which the boundary conditions can be either unknown or rapidly changing. Moreover, for experimental validation the need to control few well defined geometrical parameters is attractive. In addition, an analytical expression of the sensitivity of the modeled shape (or an associated geometrical parameter) to an uncertainty of the imposed control parameters might be of interest for experimental setup characterization or design purposes.

Quasi-analytical ring models are considered using the minor axis $b$ as an input control parameter. The pinching 


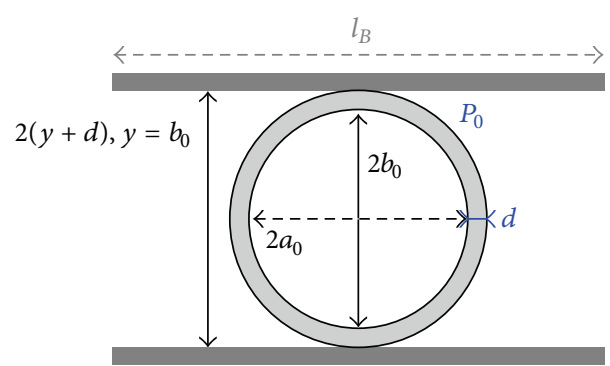

(a) Circular $b_{0}=D / 2$

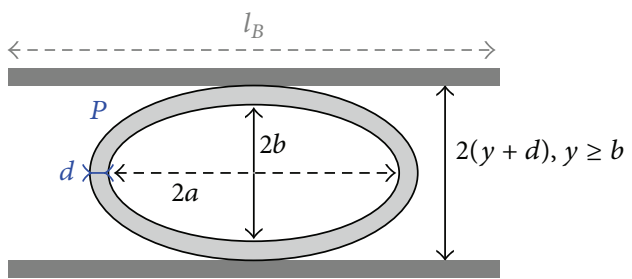

(b) Pinched $b<D / 2$

FIGURE 1: Illustration of a deformable ring (light grey) with constant wall thickness $d$ compressed between two parallel bars (dark grey) of length $0<l_{B}$. The internal central ring height yields twice the minor axis $2 b$ and the internal central width yields twice the major axis $2 a$. The parallel bars are spaced by $2(y+d)$ with $y=b$ for nonbuckled rings and $y>b$ for buckled rings. (a) Circular $\left(b_{0}=D / 2\right.$ and $\left.a_{0}=D / 2\right)$ ring with internal perimeter $P_{0}=\pi D$ (internal diameter $D$ ) and (b) elongated ring $(b<D / 2$ and $a>D / 2$ ).

degree of the ring is expressed as $1-b / b_{0}$ where the subscript ${ }_{0}$ refers to values associated with a circular ring. For a circular ring, both the minor $b_{0}$ and major $a_{0}$ axis yield half the internal diameter $D$. We consider a circular ring with internal perimeter $P_{0}=\pi D$, which is compressed between two parallel bars of length $0<l_{B}$ as illustrated in Figure 1(a). It is assumed that the ring has constant wall thickness $d$. Forcing the bars closer together (Figure 1(b)) deforms the ring so that for the ring's minor axis $0<b \leq D / 2$ holds, where $b_{0}=D / 2$ denotes the minor axis of the undeformed circular ring. The distance between the pinching bars yields $0<2(y+d) \leq$ $D+2 d$. Deforming the ring results in an elongated ring shape so that the area $A$ enveloped by the ring reduces as the bars are forced closer together. The deformed ring is assumed to be symmetrical with respect to its center lines containing the minor and major axis.

Parameterised geometrical ring models are presented in Section 2 for nonbuckled and buckled rings. Next (Section 3), a simple data-driven procedure is proposed enabling to apply the ring models in order to obtain the geometry of an elastic tube pinched between two parallel bars. A concrete case study for $l_{B} \gg 2 a$ is discussed.

\section{Geometrical Ring Models}

The internal ring shape is modeled using quasi-analytical elastic ring models which are symmetrical with respect to the center lines. The main input parameters are the minor axis $b$ and internal diameter $D$ defining the perimeter $P_{0}=\pi D$ of the unpinched circular ring. Consequently, the pinching degree $0<1-b / b_{0} \leq 1$ of the ring is assumed a known quantity. The considered ring shapes are invariant under homothetic scaling. Therefore, in the following, lengths are normalised $(\cdot)$ by the internal diameter $D$ of the circular ring so that $0<\widetilde{b}=b / D \leq 0.5, \widetilde{P}_{0}=\pi$, and $\widetilde{A}_{0}=\pi / 4$ are quantities associated with a circle with unit diameter. Note that the pinching degree is not altered by the normalisation since $1-b / b_{0}=1-\tilde{b} / \tilde{b}_{0}=1-2 \widetilde{b}$. Furthermore, since the wall thickness $\widetilde{d}$ is assumed constant, the shape of the external ring $\widetilde{R}\left(\theta, 1-b / b_{0}\right)$ is derived from the shape of the internal ring as $\widetilde{R}\left(\theta, 1-\widetilde{b} / \widetilde{b}_{0}\right)=\tilde{r}\left(\theta, 1-\widetilde{b} / \widetilde{b}_{0}\right)+\operatorname{sgn}(\widetilde{r}) \cdot \tilde{d}$, with sign function sgn and the ring expressed in polar coordinates with normalised internal radius $\widetilde{r}$, normalised external radius $\widetilde{r}$, and angle $0 \leq \theta \leq 2 \pi$. Depending on the length of the parallel pinching bars $l_{B}$ compared to the length of the major axis $a$ buckling of the elastic ring will occur $\widetilde{l}_{B} \gg 2 \widetilde{a}$ (Figure 1 ) or not $\widetilde{l}_{B} \ll 2 \widetilde{a}$ as the pinching degree $1-\tilde{b} / \widetilde{b}_{0}$ is increased. Therefore, in Section 2.1, geometrical ring models are presented which does not allow to describe buckling. In Section 2.2, a ring model is presented for which buckling of the elastic ring occurs when the pinching degree $1-\widetilde{b} / \widetilde{b}_{0}$ increases.

2.1. Geometrical Nonbuckling Ring Models: Ellipse and Stadium. The internal ring is pinched between long parallel bars so that $\widetilde{l}_{B} \gg 2 \widetilde{a}$ as depicted in Figure 1 . The ring shape is approximated as an ellipse or a stadium with rounded edges as illustrated in Figure 2.

2.1.1. Ellipse. For given minor axis $\widetilde{b}$ with $0<\widetilde{b} \leq 0.5$, the elliptic ring shape is fully determined by considering a second geometrical parameter such as perimeter $\widetilde{P}$ or major axis $\tilde{a}$.

When the major axis $\tilde{a}$ and minor axis $\tilde{b}$ are known the elliptic ring shape in polar coordinates yields $[12,13]$

$$
\tilde{r}_{\tilde{a}, \tilde{b}}(\theta)=\sqrt{\frac{\tilde{b}^{2}}{1-e^{2} \cos ^{2}(\theta)}} \quad \text { with } e=\sqrt{\left(1-\left(\frac{\tilde{b}}{\tilde{a}}\right)^{2}\right)} \text {, }
$$

where $\theta \in[0,2 \pi]$ and eccentricity $e \leq 1$. The perimeter is then obtained from the expression of Ramanujan [14]:

$$
\widetilde{P}_{\widetilde{a}, \tilde{b}}=\pi[3(\widetilde{a}+\widetilde{b})-\sqrt{(\widetilde{a}+3 \widetilde{b})(3 \widetilde{a}+\widetilde{b})}]
$$

and the area yields $\widetilde{A}_{\tilde{a}, \tilde{b}}=\pi \widetilde{b} \widetilde{a}$ with $\widetilde{A}=A / D^{2}$. The sensitivity of the radius, perimeter, and area to a variation of the imposed parameters $\Delta \widetilde{b}$ and $\Delta \widetilde{a}$ yields

$$
\begin{aligned}
& \Delta \widetilde{r}_{\tilde{a}, \tilde{b}}(\theta)=\left|\frac{\tilde{b}^{3} \cos ^{2} \theta}{\tilde{a}^{3}\left(1-e^{2} \cos ^{2}(\theta)\right)^{3 / 2}}\right| \Delta \widetilde{a} \\
&+\left|\frac{1-\cos ^{2} \theta}{\left(1-e^{2} \cos ^{2}(\theta)\right)^{3 / 2}}\right| \Delta \widetilde{b}, \\
& \Delta \widetilde{P}_{\tilde{a}, \tilde{b}}=\left|-\pi\left(\frac{6 \tilde{a}+10 \tilde{b}}{2 \sqrt{(\tilde{a}+3 \tilde{b})(3 \tilde{a}+\tilde{b})}}-3\right)\right| \Delta \tilde{a}
\end{aligned}
$$




$$
\begin{aligned}
& +\left|-\pi\left(\frac{10 \widetilde{a}+6 \tilde{b}}{2 \sqrt{(\widetilde{a}+3 \tilde{b})(3 \tilde{a}+\widetilde{b})}}-3\right)\right| \Delta \widetilde{b}, \\
& \Delta \widetilde{A}_{\widetilde{a}, \tilde{b}}=\pi \widetilde{a} \Delta \widetilde{b}+\pi \widetilde{b} \Delta \widetilde{a} .
\end{aligned}
$$

When the perimeter is used as a second model input parameter instead of the major axis $\widetilde{a}$, we assume that its value is conserved when the elastic ring deforms so that $\widetilde{P}_{\vec{b}}=\pi$ holds for all pinching degrees $1-\widetilde{b} / \widetilde{b}_{0}$. The elliptic ring shape in polar coordinates for $\theta \in[0,2 \pi]$ is again of the form (1) for which eccentricity $e$ is only a function of $\widetilde{b}$ since using (2) it follows that

$$
\begin{aligned}
\tilde{r}_{\tilde{b}}(\theta)=\sqrt{\frac{\widetilde{b}^{2}}{1-\left(1-\widetilde{b}^{2} / \widetilde{a}_{\tilde{b}}^{2}\right) \cos ^{2}(\theta)}} \\
\quad \text { with } \widetilde{a}_{\tilde{b}}=\frac{\sqrt{-20 \widetilde{b}^{2}+12 \tilde{b}+3}}{6}-\frac{2 \widetilde{b}}{3}+\frac{1}{2} .
\end{aligned}
$$

The area $\widetilde{A}$ yields again $\widetilde{A}_{\widetilde{b}}=\pi \widetilde{b} \widetilde{a}_{\tilde{b}}$ or

$$
\widetilde{A}_{\tilde{b}}=\pi \widetilde{b}\left(\frac{\sqrt{-20 \widetilde{b}^{2}+12 \widetilde{b}+3}}{6}-\frac{2 \widetilde{b}}{3}+\frac{1}{2}\right) .
$$

The sensitivity of the radius to a variation of the imposed parameter $\Delta \widetilde{b}$ yields

$$
\begin{aligned}
& \frac{\Delta \widetilde{r}_{\tilde{b}}(\theta)}{\Delta \widetilde{b}}=\frac{1}{\widetilde{r}_{\tilde{b}}(\theta)}-\frac{\widetilde{b}^{2}}{\widetilde{a}_{\tilde{b}}^{2}} \\
& \quad \frac{\cos ^{2}(\theta)}{\widetilde{r}_{\tilde{b}}^{3}(\theta)}\left(1+\frac{\widetilde{b}}{3 \widetilde{a}_{\tilde{b}}}\left[\frac{10 \widetilde{b}-3}{\sqrt{-20 \widetilde{b}^{2}+12 \widetilde{b}+3}}+2\right]\right),
\end{aligned}
$$

where the last term expresses the sensitivity of the major axis to a variation of the imposed parameter $\Delta \widetilde{b}$ since

$$
\frac{\Delta \widetilde{a}}{\Delta \widetilde{b}}=\frac{-1}{3}\left(\frac{10 \widetilde{b}-3}{\sqrt{-20 \widetilde{b}^{2}+12 \widetilde{b}+3}}+2\right) .
$$

Finally, the sensitivity of the area to a variation of the imposed parameter $\Delta \widetilde{b}$ for a constant perimeter becomes

$$
\begin{aligned}
\frac{\Delta \widetilde{A}_{\widetilde{b}}}{\Delta \widetilde{b}}= & \frac{\pi}{2}-\frac{4 \pi \widetilde{b}}{3}+\frac{\pi\left(-20 \widetilde{b}^{2}+12 \widetilde{b}+3\right)^{1 / 2}}{6} \\
& +\frac{\pi \widetilde{b}\left(-20 \widetilde{b}^{2}+12 \widetilde{b}+3\right)^{-1 / 2}}{12} .
\end{aligned}
$$

2.1.2. Stadium with Rounded Edges. The stadium shape, illustrated in Figure 2, consists of two flat portions connected by circular arcs with radius $\widetilde{b}$ so that the minor axis of the stadium equals the minor axis of the ellipse. The critical angle $\varphi \in[0, \pi / 2]$ is defined by the critical distance $\widetilde{L}=$ $\widetilde{b} / \tan (\varphi) \geq 0$, which corresponds to half the extent of the flat portion in contact with the parallel pinching bars. As for the ellipse (Section 2.1.1) the stadium shape is fully determined by considering the minor axis $\widetilde{b}$ and a second geometrical parameter such as perimeter $\widetilde{P}$ or major axis $\widetilde{a}$.

Firstly, assume that the perimeter $\widetilde{P}$ is conserved when the elastic rings deform so that $\widetilde{P}_{\tilde{b}}=\pi$ holds for all pinching degrees $1-\widetilde{b} / \widetilde{b}_{0}$. In this case, the stadium ring shape $\widetilde{r}(\theta)$ with parameters $(\widetilde{b}, \widetilde{P}=\pi)$ in polar coordinates $(\widetilde{r}, \theta)$ is given as a piecewise function of $\theta \in[0,2 \pi]$ which depends explicitly on the pinching degree $1-\widetilde{b} / \widetilde{b}_{0}=1-2 \widetilde{b}$ :

$$
\tilde{r}_{\tilde{b}}(\theta)= \begin{cases}\frac{\widetilde{b}}{\sin (\theta)}, & \text { for } \varphi \leq \theta \leq \pi-\varphi, \\ \frac{\pi}{4} \cdot(1-2 \widetilde{b}) \cdot\left(\left(\frac{16}{\pi^{2}} \cdot \frac{\widetilde{b}^{2}}{(1-2 \widetilde{b})^{2}}-\sin ^{2}(\theta)\right)^{1 / 2}+\cos (\theta)\right), & \text { for }-\varphi<\theta<\varphi, \\ \frac{\pi}{4} \cdot(1-2 \widetilde{b}) \cdot\left(\left(\frac{16}{\pi^{2}} \cdot \frac{\widetilde{b}^{2}}{(1-2 \widetilde{b})^{2}}-\sin ^{2}(\theta)\right)^{1 / 2}-\cos (\theta)\right), & \text { for } \pi-\varphi<\theta<\pi+\varphi, \\ -\frac{\tilde{b}}{\sin (\theta)}, & \text { for } \pi+\varphi \leq \theta \leq 2 \pi-\varphi,\end{cases}
$$

where critical angle $\varphi_{\tilde{b}}$ and critical distance $\widetilde{L}_{\widetilde{b}}$ are a function of pinching degree $1-\widetilde{b} / \widetilde{b}_{0}=1-2 \widetilde{b}$ :

$$
\begin{aligned}
& \varphi_{\tilde{b}}=\arctan \left(\frac{4}{\pi} \cdot \frac{\widetilde{b}}{1-2 \widetilde{b}}\right), \\
& \widetilde{L}_{\widetilde{b}}=\frac{\pi(1-2 \widetilde{b})}{4} .
\end{aligned}
$$

The major axis $\widetilde{a}_{\tilde{b}}$ and area $\widetilde{A}_{\tilde{b}}$ are then expressed by a linear and, respectively, quadratic function of the imposed parameter $\widetilde{b}$ :

$$
\begin{gathered}
\widetilde{a}_{\tilde{b}}=\widetilde{b}+\frac{\pi(1-2 \widetilde{b})}{4}=\frac{\pi}{4}+\left(1-\frac{\pi}{2}\right) \widetilde{b}, \\
\widetilde{A}_{\tilde{b}}=\pi \widetilde{b}^{2}+4 \pi \widetilde{b}(1-2 \widetilde{b})=\pi \widetilde{b}(1-7 \widetilde{b}) .
\end{gathered}
$$




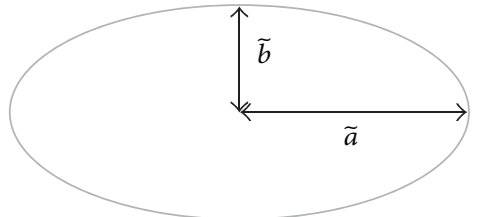

Ellipse: $(\widetilde{b}, \widetilde{P}=\pi)$ or $(\widetilde{a}, \widetilde{b})$

(a)

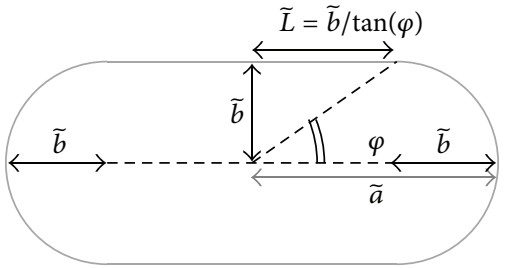

Stadium: $(\widetilde{b}, \widetilde{P}=\pi)$ or $(\widetilde{a}, \widetilde{b})$,

(b)

FIGURE 2: Overview geometrical nonbuckling ring models requiring two geometrical input parameters corresponding to a circular ring with perimeter $\widetilde{P}_{0}=\pi$ and area $\widetilde{A}_{0}=\pi / 4$ : (a) elliptic cross-section with major axis $\widetilde{a}$ and minor axis $\widetilde{b}$ indicated and (b) stadium with rounded edges with minor axis $\widetilde{b}$ and critical angle $\varphi$ indicated from which the critical distance $\widetilde{L}=\widetilde{b} / \tan (\varphi)$ can be derived.

Consequently, the sensitivity of the major axis $\Delta \widetilde{a}_{\tilde{b}}$ and area $\Delta \widetilde{A}_{\widetilde{b}}$ to a variation of the imposed parameter $\Delta \widetilde{b}$ results in, respectively, a constant and a linear function of $\widetilde{b}$ :

$$
\frac{\Delta \widetilde{a}_{\tilde{b}}}{\Delta \widetilde{b}}=1-\frac{\pi}{2},
$$

$$
\frac{\Delta \widetilde{A_{\tilde{b}}}}{\Delta \widetilde{b}}=2 \pi(2-7 \widetilde{b}),
$$

whereas the sensitivity of the radius to a variation of the experimental imposed parameter $\Delta \widetilde{b}$ is again expressed by a piecewise function of $\theta$ :

$$
\frac{\Delta \widetilde{r}_{\tilde{b}}(\theta)}{\Delta \widetilde{b}}= \begin{cases}\frac{1}{\sin (\theta)}, & \text { for } \varphi \leq \theta \leq \pi-\varphi, \\ \frac{16 \tilde{b}-2 \pi^{2}(1-2 \widetilde{b}) \sin ^{2}(\theta)}{4 \sqrt{16 \widetilde{b}^{2}-\pi^{2}(1-2 \widetilde{b})^{2} \sin ^{2}(\theta)}-\frac{\pi}{2} \cos (\theta),} & \text { for }-\varphi<\theta<\varphi, \\ \frac{16 \tilde{b}-2 \pi^{2}(1-2 \widetilde{b}) \sin ^{2}(\theta)}{4 \sqrt{16 \tilde{b}^{2}-\pi^{2}(1-2 \widetilde{b})^{2} \sin ^{2}(\theta)}+\frac{\pi}{2} \cos (\theta),} & \text { for } \pi-\varphi<\theta<\pi+\varphi, \\ -\frac{1}{\sin (\theta)}, & \text { for } \pi+\varphi \leq \theta \leq 2 \pi-\varphi,\end{cases}
$$

which $\widetilde{r}_{\tilde{b}}(9)$ depends explicitly on the pinching degree $1-\widetilde{b} / \widetilde{b}_{0}$ or $1-2 \widetilde{b}$.

Next, the major axis $\widetilde{a}$ instead of the perimeter $\widetilde{P}$ is used as a second known geometrical parameter in addition to the minor axis $\widetilde{b}$. The stadium ring shape $\widetilde{r}_{\tilde{b}, \tilde{a}}(\theta)$ with parameters $(\widetilde{b}, \widetilde{a})$ in polar coordinates $(\widetilde{r}, \theta)$ is again a piecewise function of $\theta \in[0,2 \pi]$ :

$$
\tilde{r}_{\tilde{b}, \tilde{a}}(\theta)= \begin{cases}\frac{\widetilde{b}}{\sin (\theta)}, & \text { for } \varphi \leq \theta \leq \pi-\varphi, \\ \sqrt{\widetilde{b}^{2}-\widetilde{a}^{2}\left(1-\frac{\widetilde{b}^{2}}{\widetilde{a}^{2}}\right) \sin ^{2}(\theta)}+(\widetilde{a}-\widetilde{b}) \cos (\theta), & \text { for }-\varphi<\theta<\varphi, \\ \sqrt{\widetilde{b}^{2}-\widetilde{a}^{2}\left(1-\frac{\widetilde{b}^{2}}{\widetilde{a}^{2}}\right) \sin ^{2}(\theta)}-(\widetilde{a}-\widetilde{b}) \cos (\theta), & \text { for } \pi-\varphi<\theta<\pi+\varphi, \\ -\frac{\widetilde{b}}{\sin (\theta)}, & \text { for } \pi+\varphi \leq \theta \leq 2 \pi-\varphi .\end{cases}
$$

Therefore $\widetilde{r}_{\tilde{b}, \tilde{a}}(14)$ does not depend explicitly on the pinching degree $(1-2 \widetilde{b})$, as was the case for (9), but it does depend explicitly on the critical distance $\widetilde{L}=\widetilde{a}-\widetilde{b}$ and the stadium eccentricity $e=\sqrt{1-\widetilde{b}^{2} / \widetilde{a}^{2}}$. The perimeter $\widetilde{P}_{\vec{b}, \widetilde{a}}$ and 
area $\widetilde{A}_{\widetilde{b}, \widetilde{a}}$ are, respectively, a linear and quadratic function of $\tilde{b}$ and depend linearly on $\tilde{a}$ as well:

$$
\begin{aligned}
& \widetilde{P}_{\tilde{b}, \widetilde{a}}=2 \widetilde{b}(\pi-2)+4 \widetilde{a}, \\
& \widetilde{A}_{\tilde{b}, \widetilde{a}}=4 \widetilde{a} \widetilde{b}+\widetilde{b}^{2}(\pi-4) .
\end{aligned}
$$

Consequently, the sensitivity of the perimeter $\Delta \widetilde{P}_{\widetilde{b}, \widetilde{a}}$ and area $\Delta \widetilde{A}_{\tilde{b}, \tilde{a}}$ to a variation of the input parameters $\Delta \widetilde{a}$ and
$\Delta \widetilde{b}$ is, respectively, constant and linearly dependent on $\widetilde{b}$ and $\widetilde{a}$ :

$$
\begin{aligned}
\Delta \widetilde{P}_{\tilde{b}, \tilde{a}} & =2 \pi \Delta \widetilde{b}+4 \Delta \widetilde{a}, \\
\Delta \widetilde{A}_{\widetilde{b}, \tilde{a}} & =4 \widetilde{b} \Delta \widetilde{a}+(2(\pi-4) \widetilde{b}+4 \widetilde{a}) \Delta \widetilde{b} .
\end{aligned}
$$

The sensitivity of the radius to a variation of the experimentally imposed parameter $\Delta \widetilde{b}$ is a piecewise function of $\theta$ :

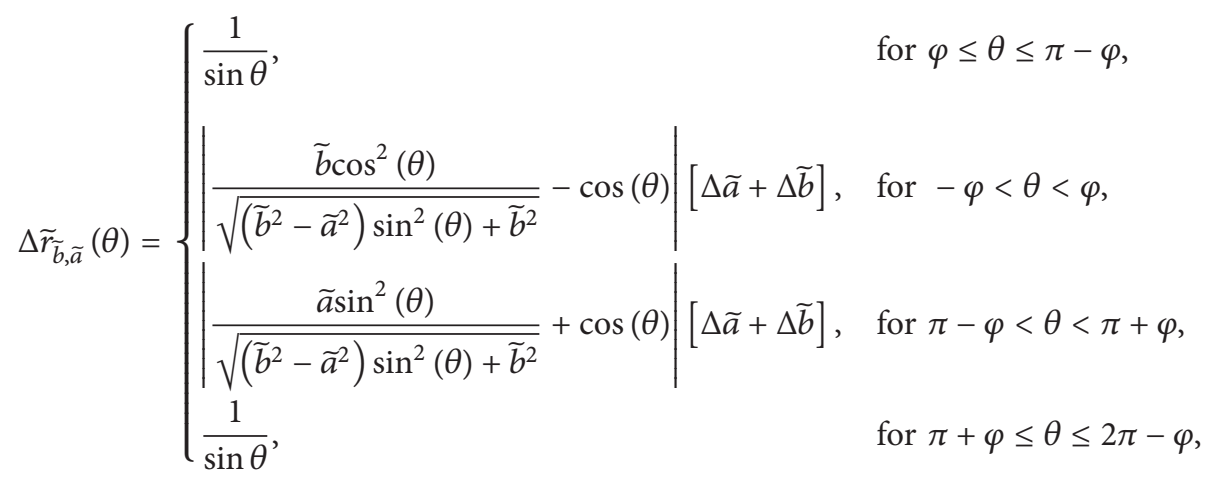

which depends explicitly on the stadium eccentricity since $\left(\tilde{b}^{2}-\tilde{a}^{2}\right)=-e^{2} \tilde{a}^{2}$.

\subsubsection{Comparison of Ellipse and Stadium Ring Models with} Perimeter $\pi$. The geometrical ellipse and stadium ring models with the assumption of constant perimeter $\widetilde{P}=\pi$ are fully determined as a function of a single parameter. We consider the minor axis $\widetilde{b}$ as a parameter since it is directly related to the pinching degree $1-\tilde{b} / b_{0}$ and therefore a control parameter for ring deformation regardless of the applied model. Single parameter models are in particular of interest to be used in combination with other analytical flow or acoustic models which require a geometrical ring (or cross-section) parameter such as major axis $\widetilde{a}$ or enveloped area $\widetilde{A}$. Therefore, in Figure 3 we consider the impact of the applied geometrical ring model in absence of buckling (ellipse or stadium) on these parameters as a function of pinching degree $1-\widetilde{b} / b_{0}$. Following (11) the normalised major axis $\tilde{a}$ of the stadium ring model increases linearly (Figure $8(c)$ ) as a function of pinching degree $1-\widetilde{b} / b_{0}$ whereas the normalised area $\widetilde{A}$ of the stadium model decreases quadratically (Figure $8(\mathrm{~d})$ ). From (4) and (5) we deduce that the ring model results in the same tendencies for major axis $\widetilde{a}$ (maximum difference between ellipse and stadium for all pinching degrees $<0.1$ ) and area $\widetilde{A}$ (maximum difference between ellipse and stadium for all pinching degrees $<0.05$ ), although the increase for $\widetilde{a}$ is not linear as is the case for the stadium model. Major axis $\tilde{a}$ of the ellipse is longer than the value of stadium model so that the area $\widetilde{A}$ enveloped by the ellipse is smaller than the area enveloped by the stadium model. The relative difference between the stadium and ellipse model for major axis $\tilde{a}$ and area $\widetilde{A}$ is further illustrated in Figure 3(c). Where the models are equivalent for both extreme pinching degrees, associated with a circular cross section in absence of pinching (1 $\widetilde{b} / b_{0}=0 \%$ and $\left.\widetilde{a}=\widetilde{b}_{0}=0.5\right)$ and a completely pinched $\operatorname{ring}\left(1-\tilde{b} / b_{0}=100 \%\right.$ and cross-section area $\left.\widetilde{A}=0\right)$, the maximum difference $(<12 \%)$ of major axis $\widetilde{a}$ between the ellipse and stadium model occurs for a pinching degree of $52 \%$ whereas the maximum difference $(<7 \%)$ between both models for area $\widetilde{A}$ occurs for a pinching degree of $66 \%$. The sensitivity of major axis $\Delta \widetilde{a}$ and area $\Delta \widetilde{A}$ to a variation of minor axis $\Delta \widetilde{b}$ is illustrated in Figures $3(\mathrm{~d})$ and $3(\mathrm{e})$. It is seen that $\Delta \tilde{a} \leq \Delta \widetilde{b}$ so that for both the ellipse and stadium $\tilde{a}$ varies less than $\widetilde{b}$ (maximum difference for all pinching degrees between ellipse and stadium $<0.8$ ). For the stadium, the variation of $\tilde{a}$ is independent from the pinching degree 1$\tilde{b} / b_{0}$ following (12). For the ellipse, $\tilde{a}$ becomes less sensitive to a variation of $\widetilde{b}$ as the pinching degree increases as expressed in (7). For small pinching degrees the impact of a variation of $\widetilde{b}$ on the area $\widetilde{A}$ is small $(\Delta \widetilde{A} \leq \Delta \widetilde{b}$ for pinching degrees $\leq 17 \%$ for the ellipse and $\leq 32 \%$ for the stadium). As the pinching degree increases the impact of a variation of $\Delta \widetilde{b}$ on the area becomes more important. In general, the impact of a variation of $\tilde{b}$ on the area is of the same order of magnitude for the ellipse and the stadium (maximum difference for all pinching degrees $<0.7$ ). Nevertheless, the area using the stadium ring is less sensitive to a variation $\Delta \widetilde{b}$ than when an ellipse is used for pinching degrees $\leq 60 \%$; for pinching degrees $>60 \%$ the opposite holds. The ellipse and stadium ring shape $\widetilde{r}(\theta)$ and associated sensitivity to a variation of minor axis $\Delta \widetilde{r}(\theta)$ are illustrated in Figure 4(a) for a pinching degree of $50 \%$. As expressed in (6) and in (13) the sensitivity depends on the angle so that it is of interest to consider a 


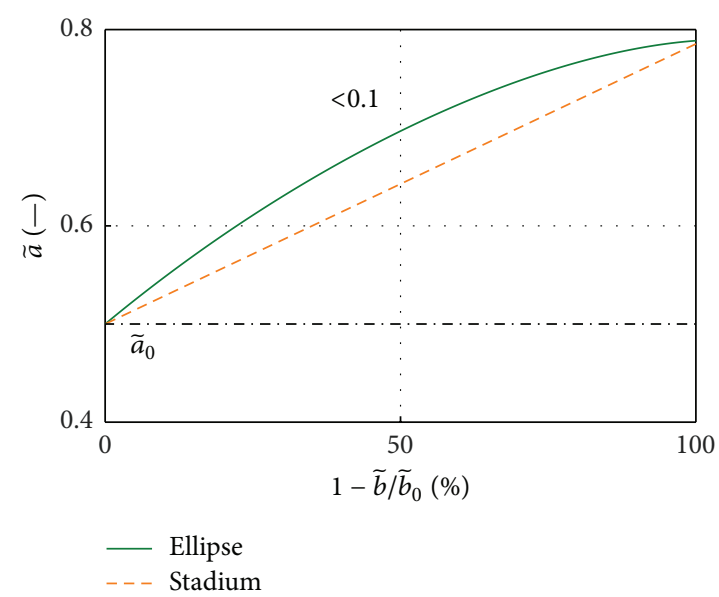

(a) $\tilde{a}$

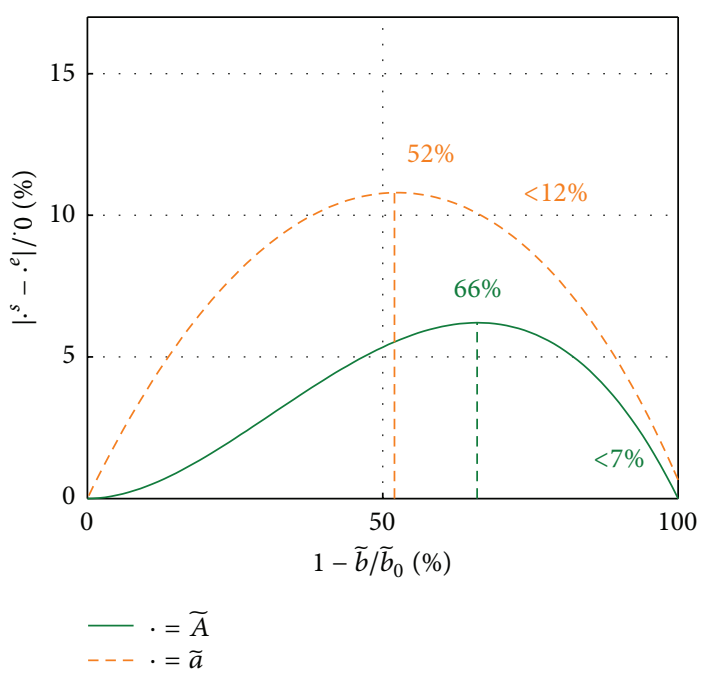

(c) $\left|\widetilde{a}^{s}-\widetilde{a}^{e}\right| / \widetilde{a}_{0}$ and $\left|\widetilde{A}^{s}-\widetilde{A}^{e}\right| / \widetilde{A}_{0}$

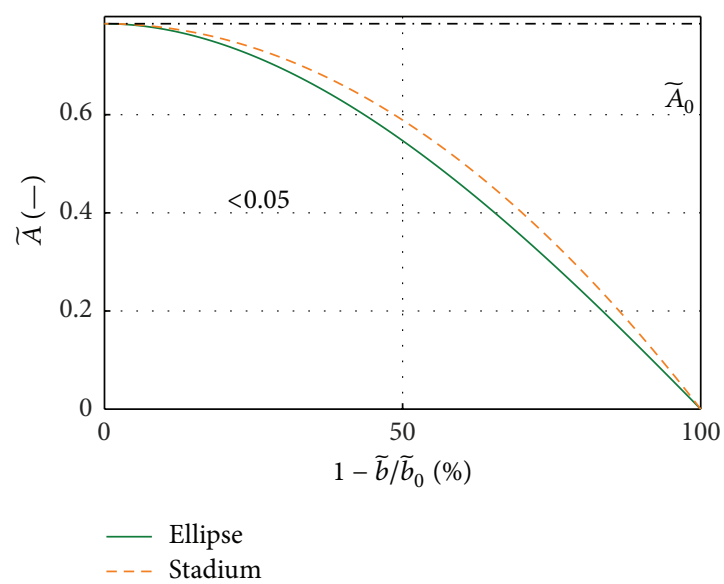

(b) $\widetilde{A}$

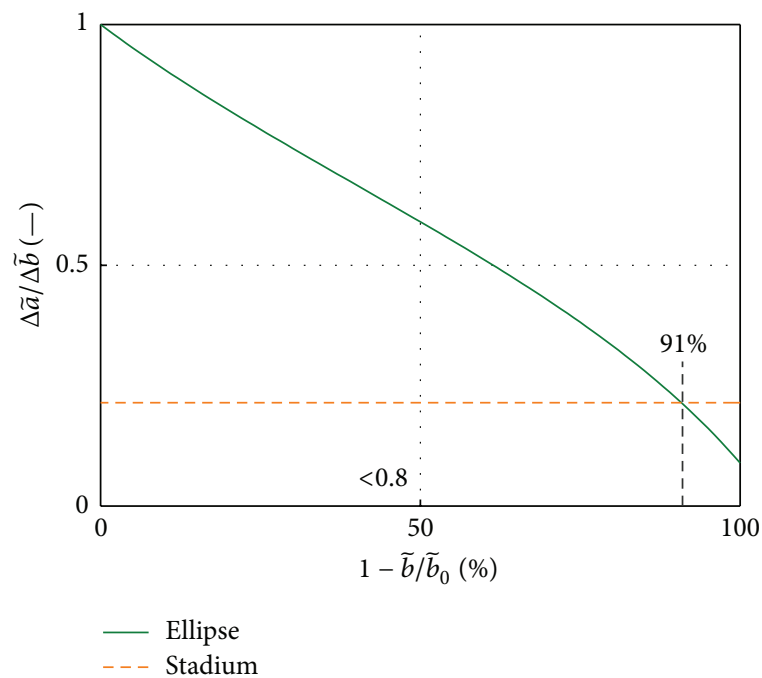

(d) $\Delta \tilde{a} / \Delta \widetilde{b}$

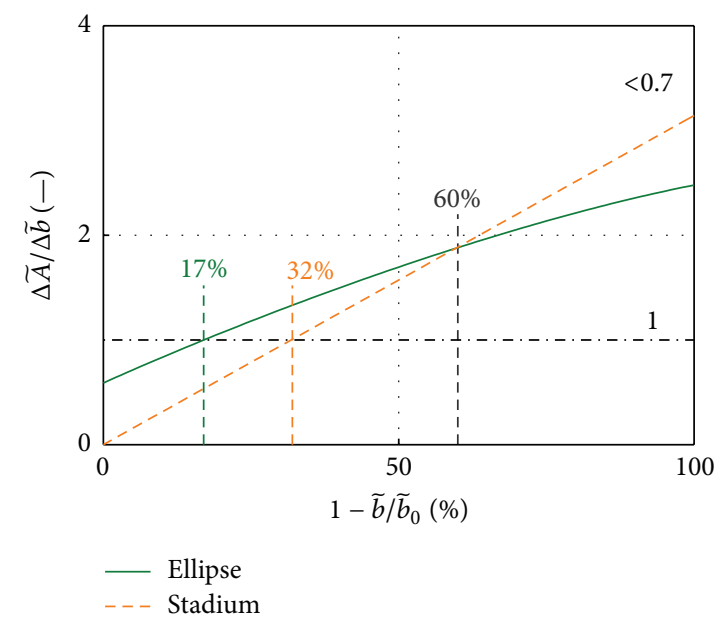

(e) $\Delta \widetilde{A} / \Delta \widetilde{b}$

FIGURE 3: Impact of applied geometrical ring models in absence of buckling, ellipse (full line in ((a), (b), (d), and (e)) and subscript $e$ in (c)) and stadium (dashed line in ((a), (b), (d), and (e)) and subscript $s$ in (c)), on normalised major axis $\widetilde{a}$ and normalised area $\widetilde{A}$ as a function of pinching degree $1-\widetilde{b} / b_{0}$ with the maximum difference indicated on each subfigure (<): (a) major axis $\widetilde{a}$ and $\widetilde{a}_{0}=0.5$ (thin dash-dot line), (b) area $\widetilde{A}$ and $\widetilde{A}_{0}=\pi / 4$ (thin dash-dot line), (c) difference between stadium model (subscript $e$ ) and ellipse (subscript $s$ ) relative to the circular value for major axis $\widetilde{a}$ (with a maximum difference for pinching degree 52\%) and area $\widetilde{A}$ (with a maximum difference for pinching degree $66 \%$ ), (d) sensitivity of major axis $\Delta \widetilde{a}$ to a variation of minor axis $\Delta \widetilde{b}$ (switch of most sensitive model for pinching degree $91 \%$ ), and (e) sensitivity of area $\Delta \widetilde{A}$ to a variation of minor axis $\Delta \widetilde{b}$ (switch of most sensitive model for pinching degree $60 \%$ ). The maximum difference is indicated as $<\cdot$. 

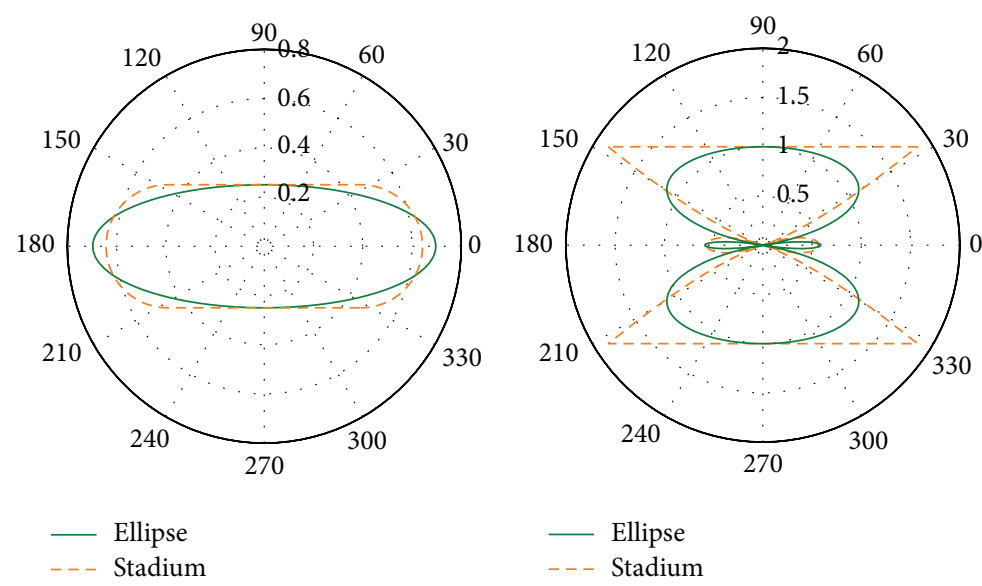

(a) Ring shape $\tilde{r}(\theta)$ (left) and sensitivity $\Delta \widetilde{r}(\theta) / \Delta \widetilde{b}$ (right) for $1-\widetilde{b} / \widetilde{b}_{0}=50 \%$

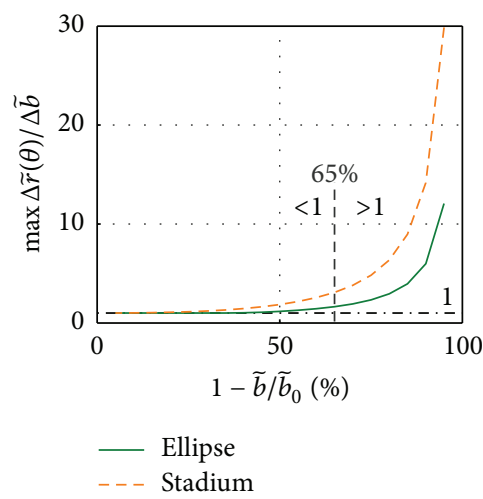

(b) $\max (\Delta \widetilde{r}(\theta)) / \Delta \widetilde{b}$

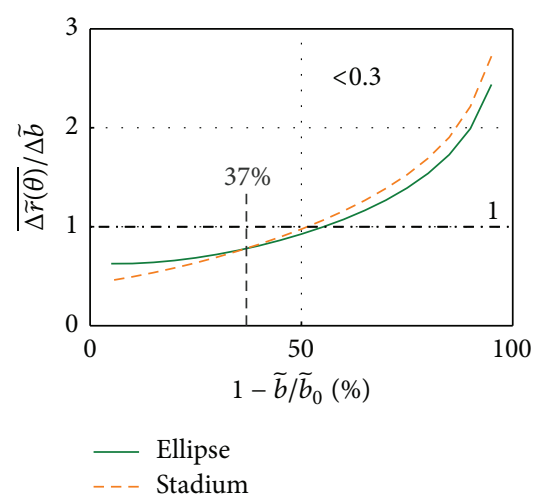

(c) $\overline{\Delta \widetilde{r}(\theta)} / \Delta \tilde{b}$

FIGURE 4: Illustration of overall sensitivity $\Delta \widetilde{r}(\theta)$ of elliptic (full line) and stadium (dashed line) ring shape $\widetilde{r}(\theta)$ to a variation of the minor axis $\Delta \widetilde{b}$ as a function of pinching degree $1-\tilde{b} / b_{0}$ : (a) example for pinching degree $50 \%$, (b) maximum value of $\Delta \widetilde{r}(\theta)$, and (c) mean sensitivity $\overline{\Delta \widetilde{r}(\theta)}$.

global measure of the ring shape to a variation of $\widetilde{b}$. Therefore, the maximum value of $\Delta \widetilde{r}(\theta)$ and mean sensitivity $\overline{\Delta \widetilde{r}(\theta)}$ for the entire ring as a function of pinching degree $1-\widetilde{b} / b_{0}$ are plotted in Figures $4(\mathrm{~b})$ and $4(\mathrm{c})$, respectively. In contrast to the sensitivity of $\widetilde{a}$, which decreases or remains constant for increasing pinching degree as shown in Figure 3(d), both global measures indicate that the overall sensitivity of the shape increases with the pinching degree. Whereas the mean sensitivity increase remains limited $(<3)$, the maximum sensitivity increase is more important since it yields up to $>5$ times the variation imposed on $\Delta \widetilde{b}$ for both the ellipse and the stadium. As was observed for the area $A$ and major axis $\tilde{a}$ the stadium ring model is more sensitive than the elliptic ring model to a variation of $\tilde{b}$ for severe constriction degrees $(\geq 60 \%)$. The observed maximum difference $(>1$ for pinching degrees $>65 \%$ ) is more of less averaged out since the difference in mean sensitivity between both ring models remains small $(<0.3)$ regardless of the pinching degree.

2.2. Buckling Ring Model: Peanut. In Section 2.1, two geometrical ring models with low computational cost were considered. Both models reduced to a single parameter ring model of the minor axis $\tilde{b}$ when applying the assumption of conservation of the perimeter $\widetilde{P}$. In this section, a quasianalytical elastic peanut ring models is outlined. Analytical elastic ring models are expected to become pertinent when the length of the parallel pinching bars (see Figure 1) reduces $\left(l_{B}<2 a\right)$ so that the loading becomes axial and buckling is likely to occur $[4,6]$.

2.2.1. Peanut. Each quarter of the peanut ring with center $\widetilde{o}$ consists of two arc portions as schematised in Figure 5. The peanut ring is then expressed as a function of the radius $\tilde{\rho}$ of the outer arc (with origin $\widetilde{o}_{1}$ ) and $\xi$ twice the angle spanned by the inner arc (with origin $\widetilde{o}_{2}$ ) so that the peanut parameter set is $(\widetilde{\rho}, \xi)$. The peanut ring model is suitable to approximate nonbuckled $(\widetilde{b} \geq \tilde{\rho})$ and buckled $(\widetilde{b}<\tilde{\rho}$, second mode) elastic rings. The assumption of conservation of perimeter is made so that the normalized perimeter of the total peanut equals the perimeter of the circular tube $\widetilde{P}=\pi$ and consequently the quarter peanut has a constant length $\pi / 4$. The total curvature of each quarter peanut yields $\pi / 2$. The minor axis $\widetilde{b} \leq 0.5$ and major axis $\tilde{a} \geq 0.5$ of the nonbuckled 


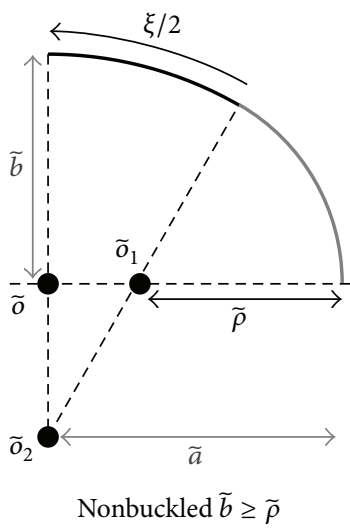

(a)

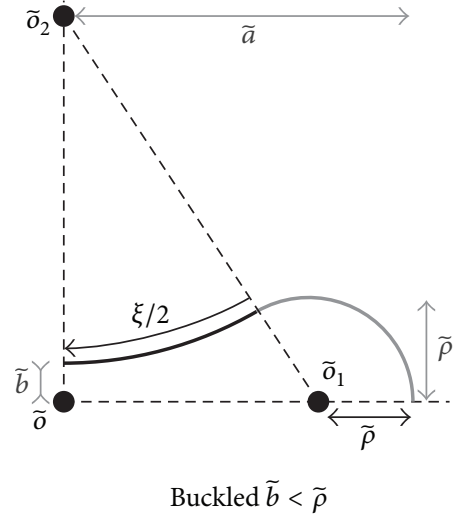

(b)

FIGURE 5: Illustration of nonbuckled ((a), inner arc curvature is positive) and buckled ((b), inner arc curvature is negative) quarters of peanut rings with center $\widetilde{o}$ and parameter set $(\widetilde{\rho}, \xi)$. Each ring consists of two arc portions: outer arc (thick gray line) with origin $\widetilde{o}_{1}$ and radius $\widetilde{\rho}$ and inner arc (thick black line) with origin $\widetilde{o}_{2}$ and angle $\xi / 2$. The major axis $\widetilde{a} \geq 0.5$ and minor axis $\widetilde{b} \leq 0.5$ of the peanut are indicated. For the nonbuckled (a) and buckled peanut (b) $\widetilde{b} \geq \widetilde{\rho}$ and $\widetilde{b}<\widetilde{\rho}$ hold, respectively.

and buckled peanuts are obtained from parameter set $(\widetilde{\rho}, \xi)$ as

$$
\begin{aligned}
\text { non-buckled: } \widetilde{b}= & \pi(1-2 \widetilde{\rho}) \frac{1-\cos (\xi / 2)}{2 \xi}+\widetilde{\rho}, \\
\widetilde{a}= & \pi(1-2 \widetilde{\rho}) \frac{\sin (\xi / 2)}{2 \xi}+\widetilde{\rho}, \\
\text { buckled: } \widetilde{b}= & \left(\frac{\pi(1-2 \widetilde{\rho})}{2 \xi}+\widetilde{\rho}\right)\left(\cos \frac{\xi}{2}-1\right) \\
& +\widetilde{\rho} \cos \frac{\xi}{2}, \\
\widetilde{a}= & \pi(1-2 \widetilde{\rho}) \frac{\sin (\xi / 2)}{2 \xi}+2 \widetilde{\rho} \sin \frac{\xi}{2} \\
& +\widetilde{\rho} .
\end{aligned}
$$

The area $\widetilde{A}(\widetilde{\rho}, \xi)$ of the total peanut is given as

$$
\begin{array}{rl}
\widetilde{A}=\pi \tilde{\rho}-\pi \tilde{\rho}^{2}+\frac{\pi^{2}}{4}(1-2 \widetilde{\rho})^{2} & f(\xi), \\
& \text { with } f(\xi)=\frac{\xi-\sin \xi}{\xi^{2}} .
\end{array}
$$

Note that for $\xi=0$ the last term in (20) is omitted since $f(\xi=0)=0$. The bending energy of the quarter peanut yields

$$
\frac{1}{4} \widetilde{\mathscr{E}}=\frac{\pi-\xi}{2 \widetilde{\rho}}+\frac{\xi^{2}}{\pi-2(\pi-\xi) \widetilde{\rho}}
$$

with $\widetilde{\mathscr{E}}(\widetilde{\rho}, \xi)$ denoting the bending energy of the total peanut. The bending energy (21) is then used to express the Lagrange functional $\mathscr{L}$ associated with the energy of deformation of the peanut ring as a function of $(\widetilde{\rho}, \xi)$ as well:

$$
\begin{aligned}
\frac{1}{\mathscr{B}} \mathscr{L}= & \frac{\pi-\xi}{\tilde{\rho}}+\frac{\xi^{2}}{\pi / 2-(\pi-\xi) \widetilde{\rho}} \\
& +\lambda\left(\widetilde{\mathscr{D}}^{2}-\frac{\pi^{2}}{16}(1-2 \widetilde{\rho})^{2}(1-\pi f(\xi))\right) \frac{1}{2 \pi},
\end{aligned}
$$

with isoperimetric difference of the quarter peanut $\widetilde{\mathscr{D}}^{2}=$ $(\pi / 4)^{2}-\pi \widetilde{A} / 4(0 \leq \widetilde{D})$, normalized pressure $\lambda=8 \mathscr{P} / \mathscr{B}$ denoting the ratio of hydrostatic pressure $\mathscr{P}$, and flexural rigidity modulus of the ring $\mathscr{B}=\Upsilon \widetilde{d}^{3} / 12\left(1-\nu^{2}\right)$ using Young's modulus $\Upsilon$, Poisson's ratio $v$, and normalised wall thickness $\widetilde{d}$.

For a given $\widetilde{A}$, it is aimed to find the peanut defined by parameter set $(\widetilde{\rho}, \xi)$ which minimises the bending energy $\widetilde{\mathscr{E}}$. When using the normalised pressure $\lambda$ as a Lagrange multiplier $\lambda$, the constrained minimisation problem satisfies the Euler-Lagrange equations in terms of $(\widetilde{\rho}, \xi, \lambda)$ :

$$
\begin{aligned}
& 0=\frac{\pi \widetilde{\rho}-\pi / 2-2 \xi \widetilde{\rho}}{(\pi / 2-(\pi-\xi) \widetilde{\rho})^{2}}+\frac{1}{4} \lambda \widetilde{\rho}^{2} \frac{1-\pi f(\xi)}{\pi-\xi}, \\
& 0=\frac{-2}{(\pi / 2-(\pi-\xi) \widetilde{\rho})^{2}}+\frac{1}{4} \lambda \tilde{\rho} f^{\prime}(\xi), \\
& \tilde{\rho}=\frac{2}{\pi}\left(\frac{\pi}{4}-\frac{\widetilde{D}}{\sqrt{1-\pi f(\xi)}}\right),
\end{aligned}
$$

where the prime denotes the derivative with respect to $\xi$. The system (23) is then solved firstly by eliminating $\lambda(\widetilde{\rho}, \xi)$ using the first two expressions of (23)

$$
\lambda=\frac{8}{\widetilde{\rho} f^{\prime}(\xi)(\pi / 2-(\pi-\xi) \widetilde{\rho})^{2}} .
$$


The resulting expression (24) is resubstituted in (23) to find $\xi$ satisfying

$$
\begin{aligned}
0= & \frac{2}{\tilde{\rho} f^{\prime}(\xi)(\pi / 2-(\pi-\xi) \tilde{\rho})^{2}} \\
& +\frac{(\pi-\xi)(\pi \tilde{\rho}-\pi / 2-2 \xi \tilde{\rho})}{\widetilde{\rho}^{2}(1-\pi f(\xi))(\pi / 2-(\pi-\xi) \tilde{\rho})^{2}}
\end{aligned}
$$

Equation (25) is solved by substituting $\widetilde{\rho}(\xi)$ given by the last equation of (23) so that a single equation for $\xi$ is obtained, from which in turn the parameter $\tilde{\rho}$ is obtained straightforwardly.

Once $\widetilde{\rho}$ and $\xi$ are determined for given $\widetilde{A}$, the polar equation of the corresponding peanut $\tilde{r}_{\tilde{\rho}, \xi}(\theta)$ with center $\widetilde{o}$ taken as the origin is directly given as a piecewise function describing two connected arc portions with centers $\widetilde{o}_{1}$ and $\widetilde{o}_{2}$ as depicted in Figure 5:

$$
\left|\widetilde{r}_{\tilde{\rho}, \xi}(\theta)\right|= \begin{cases}\tilde{\rho}, & \theta \in\left[0, \frac{\pi-\xi}{2}\right] \text { with } o_{1}=\left(\frac{(\pi / 2-\pi \tilde{\rho})}{\xi}+2 \tilde{\rho}\right) \sin \frac{\xi}{2}, \\ \left(\frac{(\pi / 2-\pi \tilde{\rho})}{\xi}+\tilde{\rho}\right), & \theta \in\left[\frac{\pi-\xi}{2}, \frac{\pi}{2}\right] \text { with } o_{2}=\left(\frac{(\pi / 2-\pi \tilde{\rho})}{\xi}+2 \tilde{\rho}\right) \cos \frac{\xi}{2} .\end{cases}
$$
Figure 6(b).

Instead of imposing the normalised area $\widetilde{A}$, the pinching degree $1-\tilde{b} / b_{0}$ can be used as a constrain parameter using the expression of the minor axis (19). The relationship between normalised area $\widetilde{A}$ and pinching degree $1-\widetilde{b} / b_{0}$ is depicted in Figure 6(a). It is seen that the peanut is fully pinched $\left(1-\tilde{b} / b_{0}=100 \%\right)$ when the area $\widetilde{A}$ yields about $25 \%$ of $\widetilde{A}_{0}$. Pinching degrees greater than $100 \%$ result in nonphysical peanut shapes since it corresponds to a negative value of the minor axis $\widetilde{b}$ and are therefore not considered further. The onset of buckling occurs for pinching degrees greater than $54 \%$ (or areas smaller than about $70 \%$ of $\widetilde{A}_{0}$ ) so that for those pinching degrees the value of the parameter $\tilde{\rho}$ is greater than the minor axis $\widetilde{b}$ as illustrated in

$$
\left(\frac{\partial F_{j}}{\partial x_{i}}\right)=\left[\begin{array}{ccc}
\mathscr{A}_{a} & \mathscr{A}_{b} & \frac{\tilde{\rho}^{2}(1-\pi f(\xi))}{4(\pi-\xi)} \\
\mathscr{A}_{c} & \mathscr{A}_{d} & \frac{\tilde{\rho} f^{\prime}(\xi)}{4} \\
\frac{\pi^{2}}{4}(1-2 \tilde{\rho})[1-\pi f(\xi)] & \frac{\pi^{3}}{16}(1-2 \tilde{\rho})^{2} f^{\prime}(\xi) & 0
\end{array}\right],
$$

The Euler-Lagrange equations (23) can be seen as a mapping function $F:(\widetilde{\rho}, \xi, \lambda ; \widetilde{A}) \mapsto \mathbb{R}^{3}$ for which the parameter vector $x=(\tilde{\rho}, \xi, \lambda)$ is determined for each $\widetilde{A}$ by solving $F(x ; \widetilde{A})=0$. Consequently, an expression for the modulus of deformation of the peanut $\mu=d \lambda / d A$ is computed by applying the chain rule to find the derivatives $\partial x_{i} / \partial A$ and furthermore using Cramer's rule as [15]:

$$
\begin{aligned}
\mu= & \frac{1}{\operatorname{det}\left(\partial F_{j} / \partial x_{i}\right)} \\
& \cdot\left|\begin{array}{ccr}
\mathscr{A}_{a} & \mathscr{A}_{b} & 0 \\
\mathscr{A}_{c} & \mathscr{A}_{d} & 0 \\
\frac{\pi^{2}}{4}(1-2 \tilde{\rho})[1-\pi f(\xi)] & \frac{\pi^{3}}{16}(1-2 \widetilde{\rho})^{2} f^{\prime}(\xi) & \pi
\end{array}\right|,
\end{aligned}
$$

with matrix

$$
\begin{aligned}
& \mathscr{A}_{c}=\frac{-4(\pi-\xi)}{(\pi / 2-(\pi-\xi) \tilde{\rho})^{3}}+\frac{1}{4} \lambda f^{\prime}(\xi), \\
& \mathscr{A}_{d}=\frac{4 \widetilde{\rho}}{(\pi / 2-(\pi-\xi) \tilde{\rho})^{3}}+\frac{1}{4} \lambda \tilde{\rho} f^{\prime \prime}(\xi) .
\end{aligned}
$$

$$
\begin{aligned}
\mathscr{A}_{b}= & \frac{2 \tilde{\rho}^{2} \xi}{(\pi / 2-(\pi-\xi) \tilde{\rho})^{3}} \\
& +\frac{1}{4} \lambda \tilde{\rho}^{2}\left[\frac{1-\pi f(\xi)}{(\pi-\xi)^{2}}-\frac{\pi f^{\prime}(\xi)}{\pi-\xi}\right],
\end{aligned}
$$

Figure 7(a) illustrates the increase in normalised pressure $\lambda(\rho, \xi)$ when the normalised peanut area $\widetilde{A}$ is decreased from the value of a circle with unit diameter $\left(\widetilde{A}_{0}\right)$ to complete closure $(\widetilde{A}=0)$ following (24). The resulting modulus of 


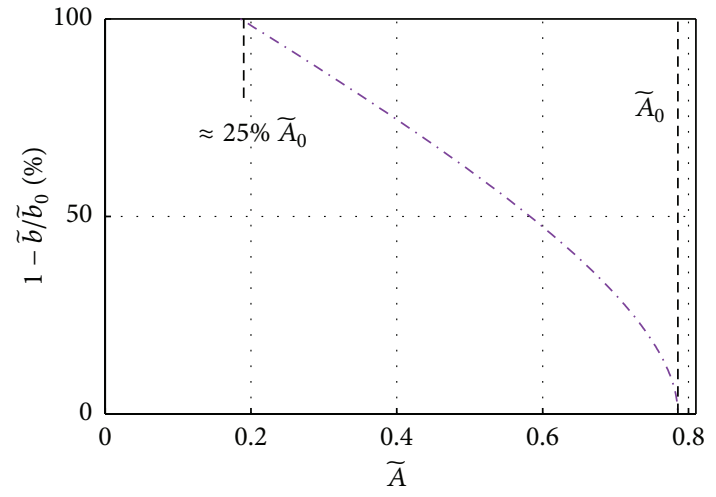

(a) $\tilde{b}(\widetilde{A})$

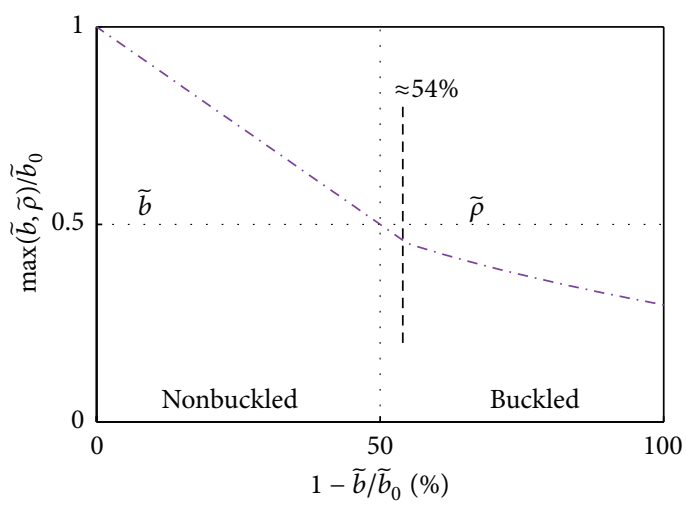

(b) $\max (\widetilde{b}, \widetilde{\rho}) / \widetilde{b}_{0}$

FIGURE 6: Illustration of peanut parameters: (a) pinching degree $1-\widetilde{b} / \widetilde{b}_{0}$ as a function of area $\widetilde{A}$ with $\widetilde{A}_{0}=\pi / 4$ (dashed line) and (b) maximum value of minor axis $\tilde{b}$ and peanut parameter $\tilde{\rho}$ as a function of pinching degree $1-\widetilde{b} / \widetilde{b}_{0}$. The shift from a nonbuckled to a buckled ring is indicated.

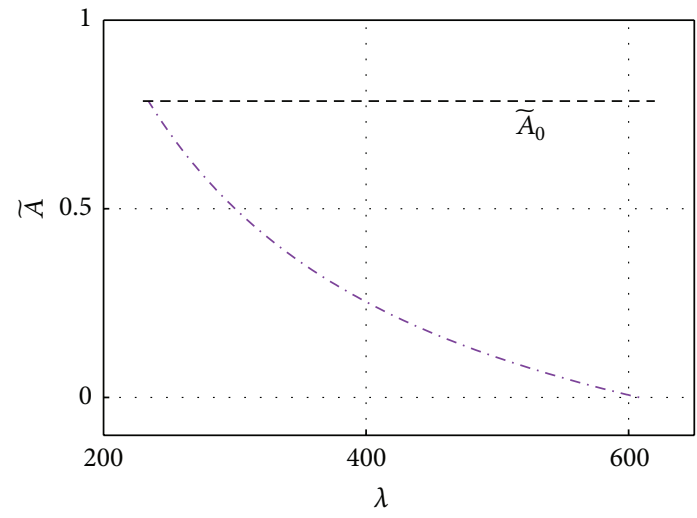

(a) $\widetilde{A}(\lambda)$

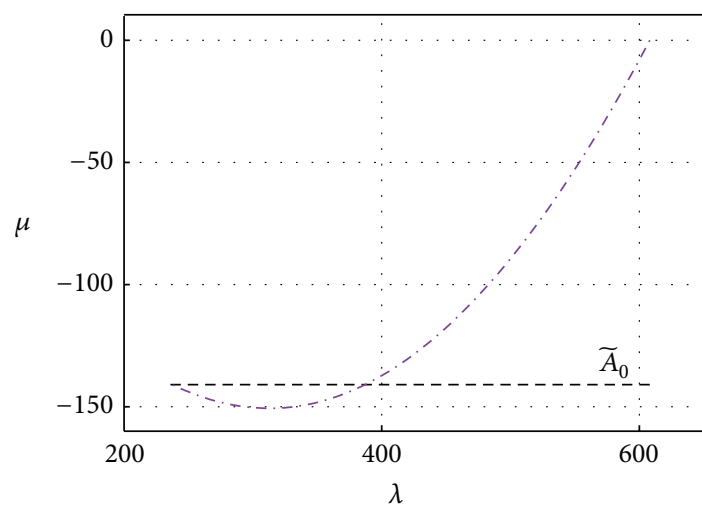

(b) $\mu(\lambda)$

Figure 7: (a) Normalised pressure $\lambda$ as a function of area $\widetilde{A}$. The dashed line corresponds to $\widetilde{A}_{0}=\pi / 4$. (b) Modulus of deformation $\mu$ as a function of normalised pressure $\lambda$. As a reference the value of the modulus for $\widetilde{A}_{0}=\pi / 4$ is indicated (dashed line).

deformation $\mu$ as a function of normalised pressure $\lambda$ is shown in Figure 7.

\subsubsection{Comparison of Peanut and Nonbuckling Ring Models} with Perimeter $\pi$. Examples of ring shapes obtained using the elliptic, stadium, and peanut ring model for pinching degrees of $30 \%$ and $80 \%$ are illustrated in Figures 8(a) and $8(\mathrm{~b})$, respectively. The resulting rings are relatively similar for $30 \%$ for which the peanut ring is nonbuckled whereas for $80 \%$ the peanut ring buckles so that the difference between the peanut shape and the other ring models becomes more pronounced. The impact of the model choice is further quantified as a function of pinching degree $1-\widetilde{b} / \widetilde{b}_{0}$ for major axis $\widetilde{a}$ (Figure $8(\mathrm{c})$ ) and enveloped area $\widetilde{A}$ (Figure $8(\mathrm{~d})$ ). It is seen that for the nonbuckled peanut shapes corresponding to pinching degrees $\leq 54 \%$ the major axis and area of the peanut yields values in the range between the ones obtained with the elliptic and stadium model. Indeed, the peanut model results in values of $\widetilde{a}$ and $\widetilde{A}$ similar to the elliptic model for low pinching degrees $(\leq 15 \%)$ and gradually evolves towards values obtained with the stadium model as the pinching degree is increased from $15 \%$ to $54 \%$. Consequently, for pinching degrees $\leq 54 \%$ the difference between the modeled ring values for $\widetilde{a}$ and $\widetilde{A}$ increases with pinching degree due to the increasing difference between the elliptic and stadium ring model which yields $<12 \%$ for $\widetilde{a}$ and $<7 \%$ for $\widetilde{A}$ (see Figure $3(\mathrm{c})$ ). For pinching degrees $>54 \%$ the peanut ring is buckled compared to the nonbuckled rings obtained with the elliptic and stadium model. As a result, the difference between modeled values of $\widetilde{a}$ and $\widetilde{A}$ obtained with the nonbuckling elliptic and stadium models reduces as the pinching degree increases whereas the difference between the nonbuckling models and the buckled peanut model increases with pinching degree up to $45 \%$ for $\widetilde{a}$ and up to $25 \%$ for $\widetilde{A}$.

\section{Application to an Elastic Tube with $l_{B} \gg 2 a$}

It is aimed to evaluate if the two-dimensional ring models outlined in the previous sections can be applied to model a three-dimensional elastic tube which is pinched somewhere 


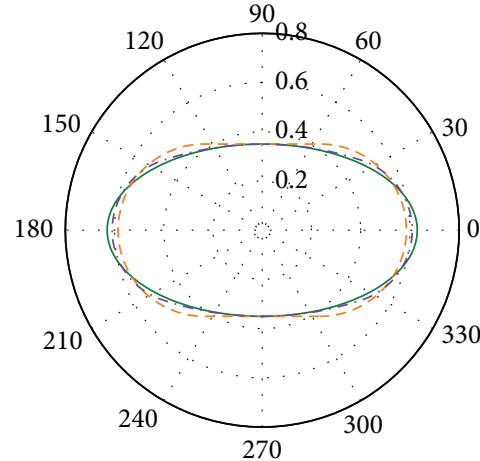

(a) $\widetilde{r}(\theta)$ for $1-\widetilde{b} / b_{0}=30 \%$

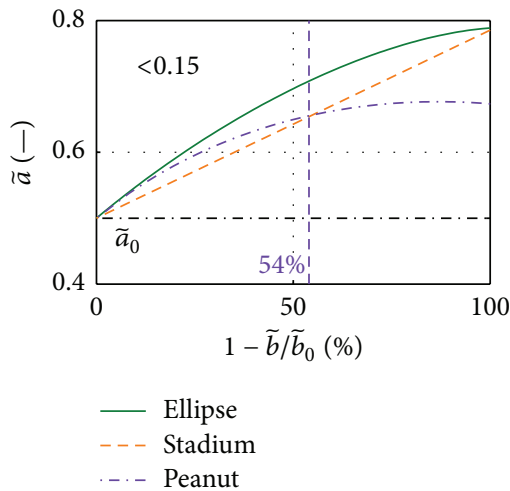

(c) $\tilde{a}\left(1-\tilde{b} / b_{0}\right)$

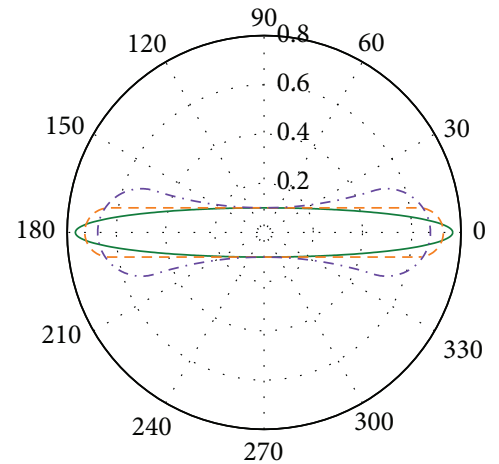

(b) $\widetilde{r}(\theta)$ for $1-\widetilde{b} / b_{0}=80 \%$

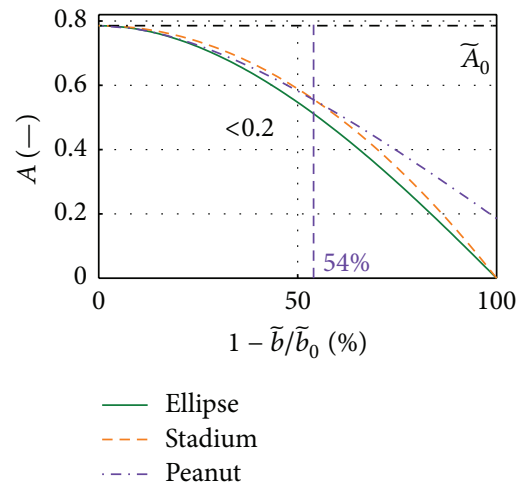

(d) $\widetilde{A}\left(1-\tilde{b} / b_{0}\right)$

FIGURE 8: Illustration of nonbuckling (ellipse, stadium) and buckling (peanut) geometrical ring models with perimeter $\pi$ as a function of pinching degree $1-\widetilde{b} / \widetilde{b}_{0}:((\mathrm{a}),(\mathrm{b}))$ ring shapes $\widetilde{r}(\theta)$ for pinching degrees of $30 \%$ and $80 \%$ and $((\mathrm{c})$, (d)) major axis $\widetilde{a}$ and area $\widetilde{A}$. As a reference $\widetilde{a}_{0}=0.5$ and $\widetilde{A}_{0}=\pi / 4$ are indicated. In addition, the threshold value of the pinching degree $(\approx 54 \%)$ for the peanut between nonbuckling and buckling is depicted (see Figure 6(b)).

along the longitudinal direction using a simple data-driven procedure. The parallel pinching bars have a length larger than twice the major axis so that $l_{B} \gg 2 a$ holds regardless of the pinching degree. Consequently, it is aimed to model each cross-section as a two-dimensional nonbuckled ring with constant perimeter which is equal to the perimeter of the unpinched circular tube. Therefore, all previously discussed ring models (ellipse, stadium, and peanut) can be applied when one additional parameter, that is, local pinching degree, is known at each cross section. The longitudinal variation of the pinching degree is expressed as follows: $1-b(x) / b_{0}$ since the minor axis $b$ depends on the longitudinal dimension $x$. The tube's geometry, the gathering of geometrical data, and a simple data-driven procedure to apply the two-dimensional (2D) ring models to model a three-dimensional (3D) tube geometry are outlined in Section 3.1. The comparison of modeled and measured geometrical tube features is then presented in Section 3.2.

3.1. Extension from 2D Ring Models to 3D Tube Models: A Data-Driven Procedure. Geometrical data are acquired for a cylindrical elastic tube of length $l=184 \mathrm{~mm}$ in the longitudinal $x$-direction with wall thickness $3 \mathrm{~mm}$ and the internal diameter of the undeformed cylindrical tube yields $25 \mathrm{~mm}$. The tube is equipped with a pincer at longitudinal position $x_{c}=90 \mathrm{~mm}$. Concretely, the pincer consists of two parallel circular bars of diameter $6.4 \mathrm{~mm}$ oriented along the $y$-axis which enforces a symmetrical deformation of the tubes cross-section shape with respect to its center-plane. The elastic tube with pincer is illustrated in Figures 9(a) and 9(b).

In order to obtain geometrical data, the tube is mounted on a turning platform controlled by a step motor with accuracy $1.8^{\circ}$. At each rotational position a longitudinal profile of the tube is quantified using a calibrated line laser scan $[16,17]$. The turning platform with mounted tube and single longitudinal laser line is illustrated in Figure 9. At the position of the pincer $x_{c}$ buckling is hindered due to the condition $l_{B} \gg 2 a$. Consequently, the distance between the parallel bars yields $2\left(b_{x_{c}}+d\right)$ and the minor axis $b_{x_{c}}$ is a known quantity at the pincer position $x_{c}$. The pincher effort is expressed by the imposed pincher degree at $x=x_{c}$ defined as $1-b_{x_{c}} / b_{0}$ with $b_{0}=12.5 \mathrm{~mm}$ as before denoting the internal radius of the unpinched cylindrical tube.

Concretely, longitudinal profiles are assessed for 6 different pincher efforts $1-b_{x_{c}} / b_{0}$ in the range from $40 \%$ up to $95 \%[16,17]$. In order to model the $3 \mathrm{D}$ tube using the $2 \mathrm{D}$ ring models the minor axis need to be estimated for each cross section so that the required ring model parameter $1-b_{x} / b_{0}$ is a known quantity. This way the $2 \mathrm{D}$ ring models can be applied to each cross section of the $3 \mathrm{D}$ tube resulting in an elliptic, 


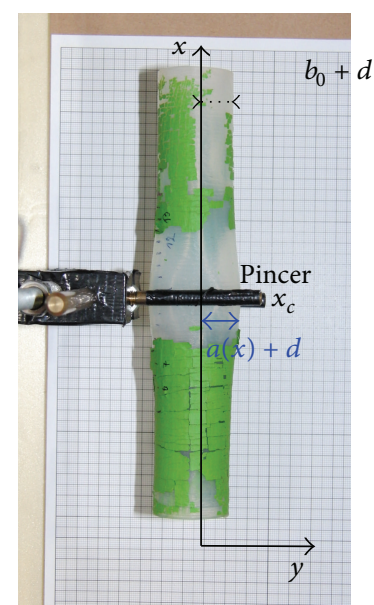

(a) Major axis $a(x)$, top view

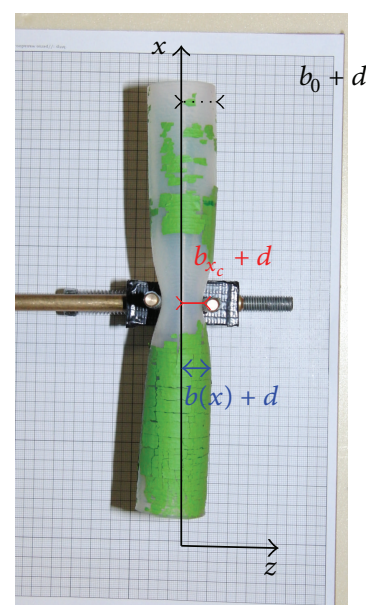

(b) Minor axis $b(x)$, side view

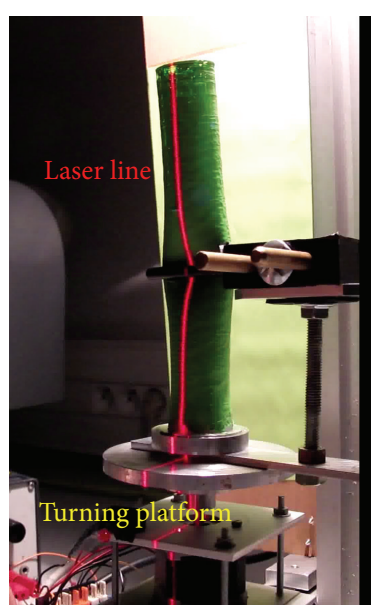

(c) Laser line for scan

FIGURE 9: Illustration of deformable tube with internal diameter $2 \times b_{0}=25 \mathrm{~mm}$ and wall thickness $d$ with pincer at longitudinal position $x=x_{c}$ and pincer effort $1-b_{x_{c}} / b_{0}$ : (a) major axis $a(x)$ ( $x y$-plane, top view), (b) minor axis ( $x z$-plane, side view), and (c) laser line from resulting in the longitudinal $x$ profile as a function of platform turning angle.

stadium, or peanut tube approximation. Therefore, firstly, the minor $(b)$ and major $(a)$ axis are extracted as a function of longitudinal position $0 \leq x \leq l$ from the measured longitudinal profiles. Next, the following parameters of the measured longitudinal profiles are extracted: the amplitude at the pincer position $x_{c}$ of the major axis $a_{x_{c}} \geq b_{0}$, the half width at half maximum amplitude of the major axis $\alpha_{a}$, and of the minor axis $\alpha_{b}$. The parameter values obtained from the 6 assessed pinching degrees are plotted in Figure 10(a) $\left(a_{x_{c}}\right)$ and in Figure 10(b) $\left(\alpha_{a, b}\right)$. Polynomial fits are applied to the measured parameters in order to estimate the parameter values for pinching degrees in the range from $40 \%$ up to $95 \%$. Concretely, a linear fit is used to estimate the maximum amplitude $a_{x_{c}}$ as a function of pinching degree $1-b_{x_{c}} / b_{0}$ (coefficient of determination $R^{2}>0.99$ ). A second order polynomial fit is used to approximate the half width at half maximum amplitude of the major axis $\alpha_{a}$ (coefficient of determination $R^{2}>0.72$ ) and the half width at half maximum amplitude of the minor axis $\alpha_{b}$ (coefficient of determination $R^{2}>0.81$ ) along the longitudinal direction as a function of pinching degree in the range from $40 \%$ up to $95 \%$. The polynomial parameter fits are shown in Figures 10(a) $\left(a_{x_{c}}\right)$ and 10(b) $\left(\alpha_{a, b}\right)$.

Using the polynomial parameter fits for $a_{x_{c}}, \alpha_{a}$, and $\alpha_{b}$ as a function of pinching effort $1-b_{x_{c}} / b_{0}$ illustrated in Figure 10, the longitudinal minor $b(x)$ and major $a(x)$ axis are subsequently approximated by symmetrical peak functions centered around the position of the pincer $x_{c}$ and function of the applied pincer effort $1-b_{x_{c}} / b_{0}$ :

$$
\begin{aligned}
& a_{\left(x_{c}, a_{x_{c}}, \alpha_{a}\right)}(x)=b_{0}+b_{0} \cdot\left(\frac{a_{x_{c}}}{b_{0}}-1\right) \\
& \cdot \exp \left(\frac{-\ln 2 \cdot\left(x-x_{c}\right)^{2}}{\alpha_{a}^{2}}\right) \\
& \text { with } a_{x_{c}}\left(b_{x_{c}}\right) \text { and } \alpha_{a}\left(b_{x_{c}}\right),
\end{aligned}
$$

$$
\begin{aligned}
b_{\left(x_{c}, b_{x_{c}}, \alpha_{b}\right)}(x)= & b_{0}-b_{0} \cdot\left(1-\frac{b_{x_{c}}}{b_{0}}\right) \\
& \cdot\left(\frac{\left(x-x_{c}\right)^{2}}{\alpha_{b}^{2}}+1\right)^{-1}
\end{aligned}
$$

with $\alpha_{b}\left(b_{x_{c}}\right)$.

An example of the measured and estimated $a(x)$ and $b(x)$ using the data-driven procedure to obtain the parameters needed to apply (31) is illustrated in Figure 10(c). It is seen that the tube deforms symmetrically around the pincer position over a total length of $8 \times b_{0}$ which corresponds to 4 times the internal diameter of the circular tube. Following this simple procedure, the considered $2 \mathrm{D}$ ring models can be applied to each cross section of the tube for pinching efforts in the range $40 \%<1-b_{x_{c}} / b_{0}<95 \%$ using (31) and the assumption of a constant perimeter $2 \pi b_{0}$.

3.2. Ring Model Selection: Comparison of Modeled and Measured Data Features. The simple data-driven procedure presented in Section 3.1 is applied to the tube for all 6 experimentally assessed pinching efforts $1-b_{x_{c}} / b_{0}$ in order to comment on the relevance of the geometrical ring models to estimate the tube geometry for different pinching degrees and the potential impact of the model choice between the elliptic, stadium, and peanut ring models.

We are particularly interested in validating the longitudinal major axis $a(x)$ since, as argued in the introduction, this parameter impacts for instance physical phenomena related to wave propagation. Figure 11(a) presents the modeled and measured values of $a_{x_{c}}\left(1-b_{x_{c}} / b_{0}\right)$ for all of the considered ring models for pinching efforts in the range from $40 \%$ up to $95 \%$. The linear increase of $a_{x_{c}}$ with pinching effort $1-b_{x_{c}} / b_{0}$ characterizing the stadium model reflects the experimentally observed tendency. In particular, the rate of increase is well represented when using the stadium model whereas the offset 

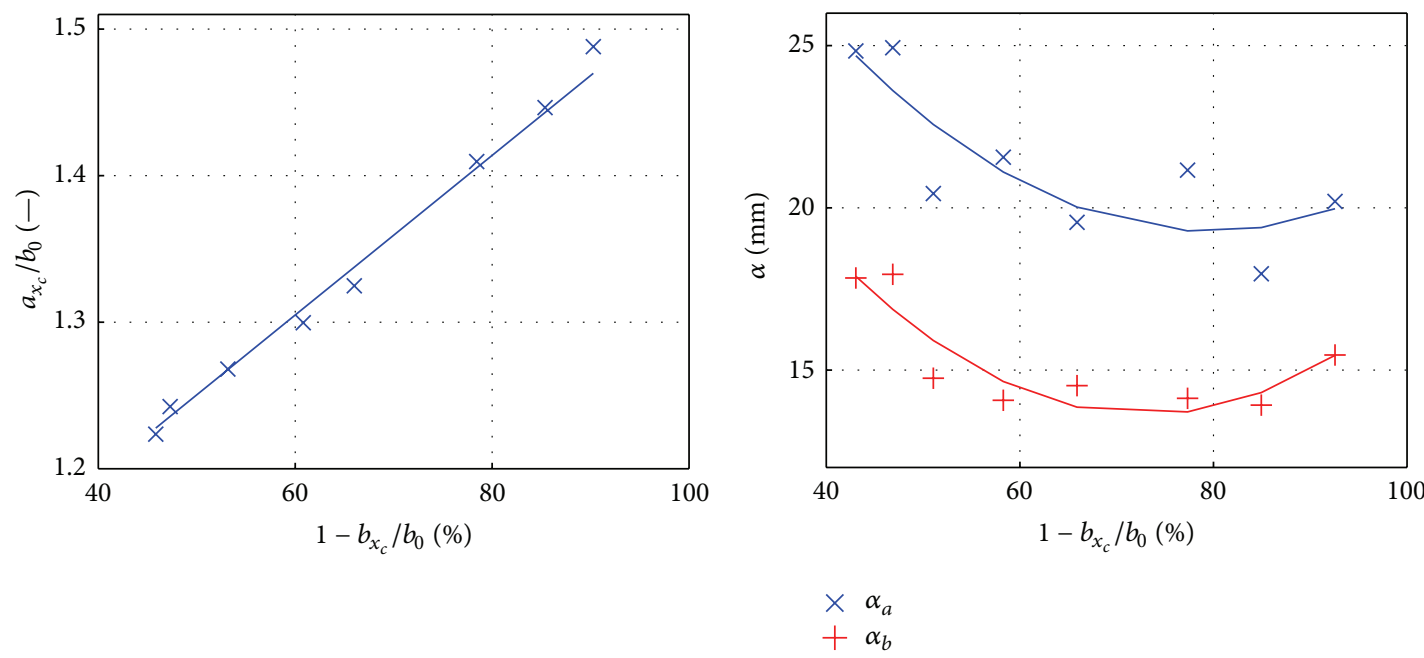

(a) $a_{x_{c}}$

(b) $\alpha_{a}, \alpha_{b}$

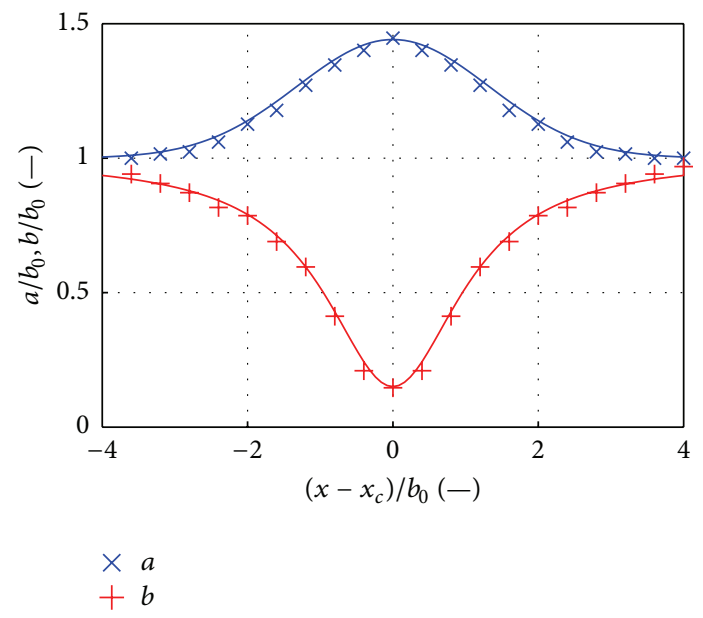

(c) $a(x)$ and $b(x)$ for $94 \%$

FIGURE 10: Fitting of symmetrical peak function parameters to experimentally measured (symbols) pincher effort $1-b_{x_{c}} / b_{0}$ [\%]: (a) linear fit (full line) to major axis maximum $a_{x_{c}}(\times)$ and (b) second order polynomial fits (full lines) to half width at half maximum for major axis $\alpha_{a}$ $(\times)$ and for minor axis $\alpha_{b}(+)$ and (c) example of measured (symbols) and peak function approximation (full lines) major $a(x)(\times)$ and minor axis $b(x)(+)$ for pincher effort $1-b_{x_{c}} / b_{0}$ of $94 \%$.

is overestimated so that $a_{x}$ is slightly (less than $5 \%$ of $b_{0}$ ) overestimated for all pinching efforts. The elliptic model results in a much more severe overestimation (up to $20 \%$ of $b_{0}$ ) for all pinching degrees so that solely on the estimation of $a_{x}$ the elliptic model can be rejected. The stadium model does not exhibit the experimentally linear increase of $a_{x_{c}}$ with pinching effort. Nevertheless, for pinching efforts smaller than $70 \%$ the accuracy of estimated values of $a_{x_{c}}$ is of the same order of magnitude as obtained using the peanut model. For pinching efforts greater than $54 \%$ buckling starts to occur which results in a severe underestimation (up to $20 \%$ of $b_{0}$ ) of $a_{x_{c}}$. Consequently, solely based on the estimation of $a_{x_{c}}$, it can be argued that the ring model pick order is stadium, peanut, and elliptic. In general, the discrepancy between the modeled longitudinal $x$ profile and the measured profile along the corresponding laser lines (such as the one depicted in Figure $9(\mathrm{c})$ ) is less than $7 \%$ of $b_{0}$ (or less than $4 \%$ of the tubes diameter) when applying the stadium ring model to each cross section with the local pinching degree computed using the peak function approximation of $b(x)$ as expressed in (31). The discrepancy between the measured and estimated longitudinal stadium profiles is illustrated in Figure 11(b) for pincher effort $1-b_{x_{c}} / b_{0}=94 \%$ using the local pinching degree associated with $b(x)$ plotted in Figure 10(c).

\section{Conclusion}

The minor axis of an elastic ring compressed between two parallel bars is directly related to the imposed pinching degree. Therefore, homothetic quasi-analytical geometrical ring models (ellipse, stadium, and peanut) are formulated for a circular ring with unit diameter as a function of pinching degree using the assumption of a constant perimeter. All three ring models reflect the same general tendencies for pinching 


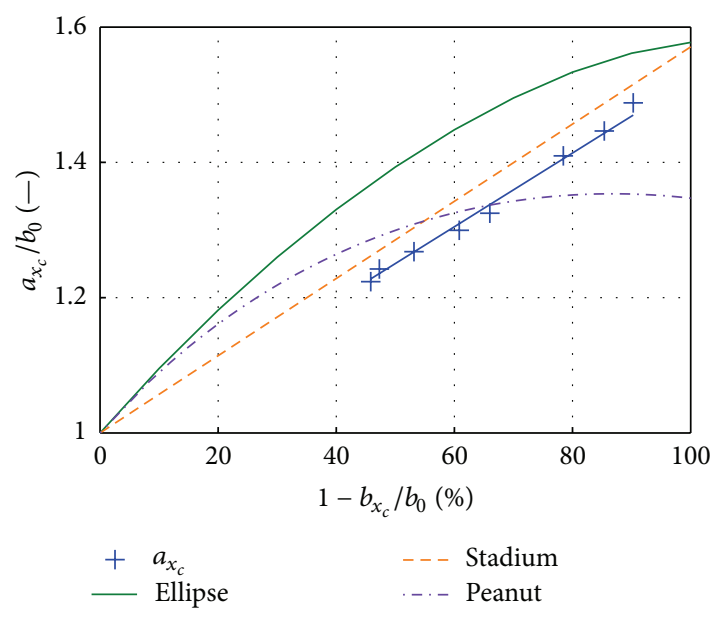

(a) $a_{x_{c}}\left(1-b_{x_{c}} / b_{0}\right) / b_{0}$

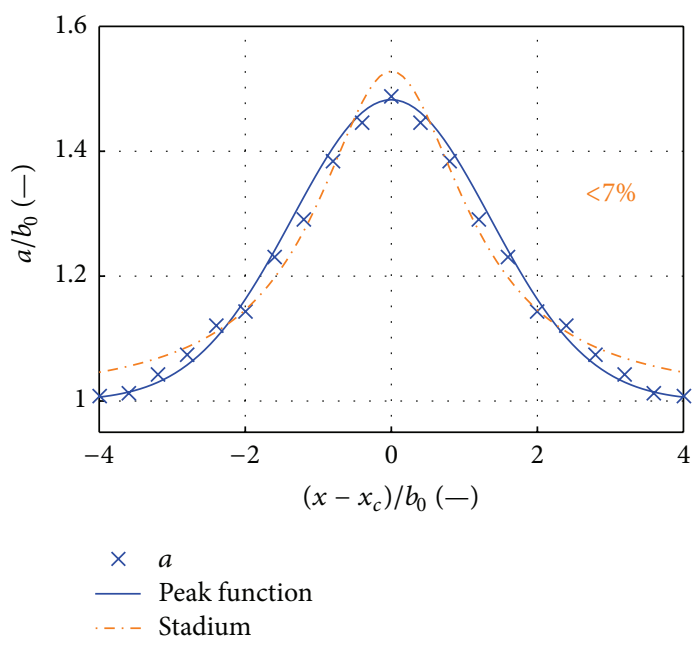

(b) $a(x) / b_{0}$

FIGURE 11: (a) Modeled and measured $a_{x_{c}} / b_{0}$ as a function of pinching effort $1-b_{x_{c}} / b_{0}$. (b) Example of measured (symbols) and stadium model (dashed line) major axis $a(x) / b_{0}$ for pincher effort $1-b_{x_{c}} / b_{0}=94 \%$ of measured $(\times)$ and peak function approximation (full line) using the local pinching degree associated with $b(x)$ plotted in Figure 10(c).

degrees up to $54 \%$. Therefore, the dynamics due to model choice for pinching degrees $\leq 54 \%$ is limited (e.g. $\leq 7 \%$ for the area and $\leq 12 \%$ for the major axis) and is determined by the difference between an elliptic and stadium ring since the peanut ring evolves gradually from elliptic $(\leq 15 \%)$ to stadium (at 54\%). For pinching degrees greater than 54\%, the peanut ring starts to buckle. As a consequence, the dynamics of geometrical features related to the model choice becomes more important (e.g. $\leq 25 \%$ for the area and $\leq 45 \%$ for the major axis).

Next, the ring models are applied to each cross section of an elastic circular tube compressed between two parallel bars for different pinching efforts between $40 \%$ and $95 \%$. A simple data-driven procedure is used to provide the local pinching degree required as an input parameter. Overall, the stadium ring model provides the best approximation of the tube with a characteristic error yielding less than $4 \%$ of the tubes diameter. Moreover, the experimentally observed linear increase of the major axis with the pinching effort is an inherent property of the stadium model. Consequently, the case study illustrates that the outlined quasi-analytical models, such as the stadium model, can provide an accurate and computational low cost approximation of the tubes geometry suitable for applications such as geometry initialisation, combination with other parameterised physical/mathematical models or experimental setup design, and validation purposes as a function of pinching degree.

\section{Conflict of Interests}

The author declares that there is no conflict of interests regarding the publication of this paper.

\section{Acknowledgments}

This work was partly supported by EU-FET Grant (EUNISON 308874). Daniel Manica and Guillaume Popovici are acknowledged for their contribution to the experiments.

\section{References}

[1] P. Kundu, Fluid Mechanics, Academic Press, London, UK, 1990.

[2] A. Pierce, Acoustics. An Introduction to Its Physical Principles and Applications, Acoustical Society of America, New York, NY, USA, 1991.

[3] S. Houliara and S. A. Karamanos, "Buckling and post-buckling of long pressurized elastic thin-walled tubes under in-plane bending," International Journal of Non-Linear Mechanics, vol. 41, no. 4, pp. 491-511, 2006.

[4] Y. Zhu, X. Y. Luo, and R. W. Ogden, "Asymmetric bifurcations of thick-walled circular cylindrical elastic tubes under axial loading and external pressure," International Journal of Solids and Structures, vol. 45, no. 11-12, pp. 3410-3429, 2008.

[5] A. Goriely, R. Vandiver, and M. Destrade, "Nonlinear Euler buckling," Proceedings of the Royal Society A: Mathematical, Physical \& Engineering Sciences, vol. 464, no. 2099, pp. 30033019, 2008.

[6] Y. Zhu, X. Y. Luo, and R. W. Ogden, "Nonlinear axisymmetric deformations of an elastic tube under external pressure," European Journal of Mechanics-A/Solids, vol. 29, no. 2, pp. 216-229, 2010.

[7] P. A. Djondjorov, V. M. Vassilev, and I. M. Mladenov, "Analytic description and explicit parametrisation of the equilibrium shapes of elastic rings and tubes under uniform hydrostatic pressure," International Journal of Mechanical Sciences, vol. 53, no. 5, pp. 355-364, 2011.

[8] S. K. Kourkoulis, C. F. Markides, and E. D. Pasiou, "A combined analytic and experimental study of the displacement field in a circular ring," Meccanica, vol. 50, no. 2, pp. 493-515, 2015.

[9] R. von Mises, "Der kritische Aubendruck zulindrischer rohre," VDI Zeitschrift, vol. 58, pp. 750-755, 1914.

[10] A. E. H. Love, The Mathematical Theory of Elasticity, Cambridge University Press, London, UK, 1927.

[11] R. Levien, “The elastica: a mathematical history," Tech. Rep., EECS Department, University of California, Berkeley, Calif, USA, 2008. 
[12] M. Abramovitz and I. A. Stegun, Handbook of Mathematical Functions, US Government Printing Office, Washington, DC, USA, 1972.

[13] B. D. Gupta, Mathematical Physics, Vikas Publishing House, New Delhi, India, 2002.

[14] S. Ramanujan, "Modular equations and approximations to $\pi$," The Quarterly Journal of Pure and Applied Mathematics, vol. 45, no. 1, pp. 350-372, 1914.

[15] F. Liu and A. Treibergs, "On the compression of elastic tubes," Tech. Rep., 2011, http://www.math.utah.edu/ treiberg/LT2006 .pdf.

[16] D. Manica, "Realisation, calibration and implementation of a laser scan for object reconstruction," Tech. Rep., Grenoble Universities, Grenoble, France, 2013.

[17] G. Popovici, "Modeling of deformed tube geometries from reconstructed and measured data," Tech. Rep., Grenoble Universities, Grenoble, France, 2014. 


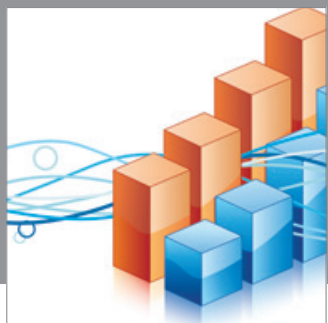

Advances in

Operations Research

mansans

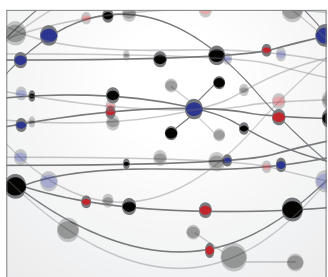

The Scientific World Journal
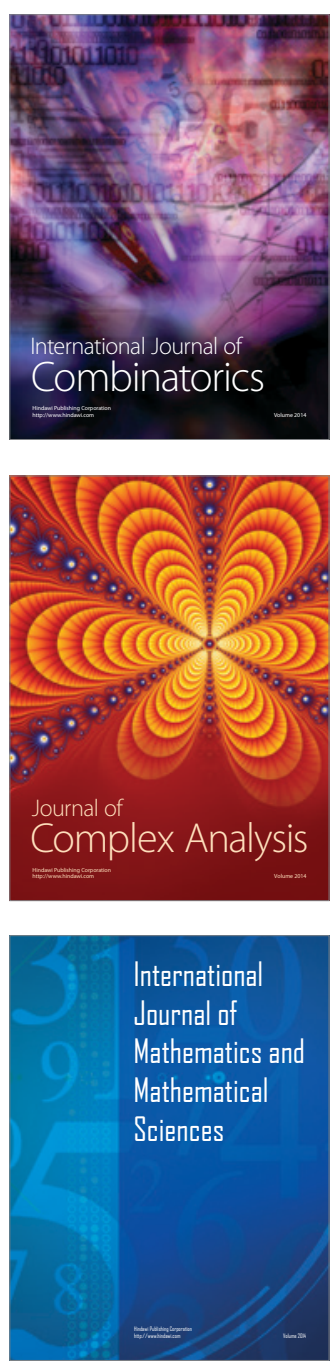
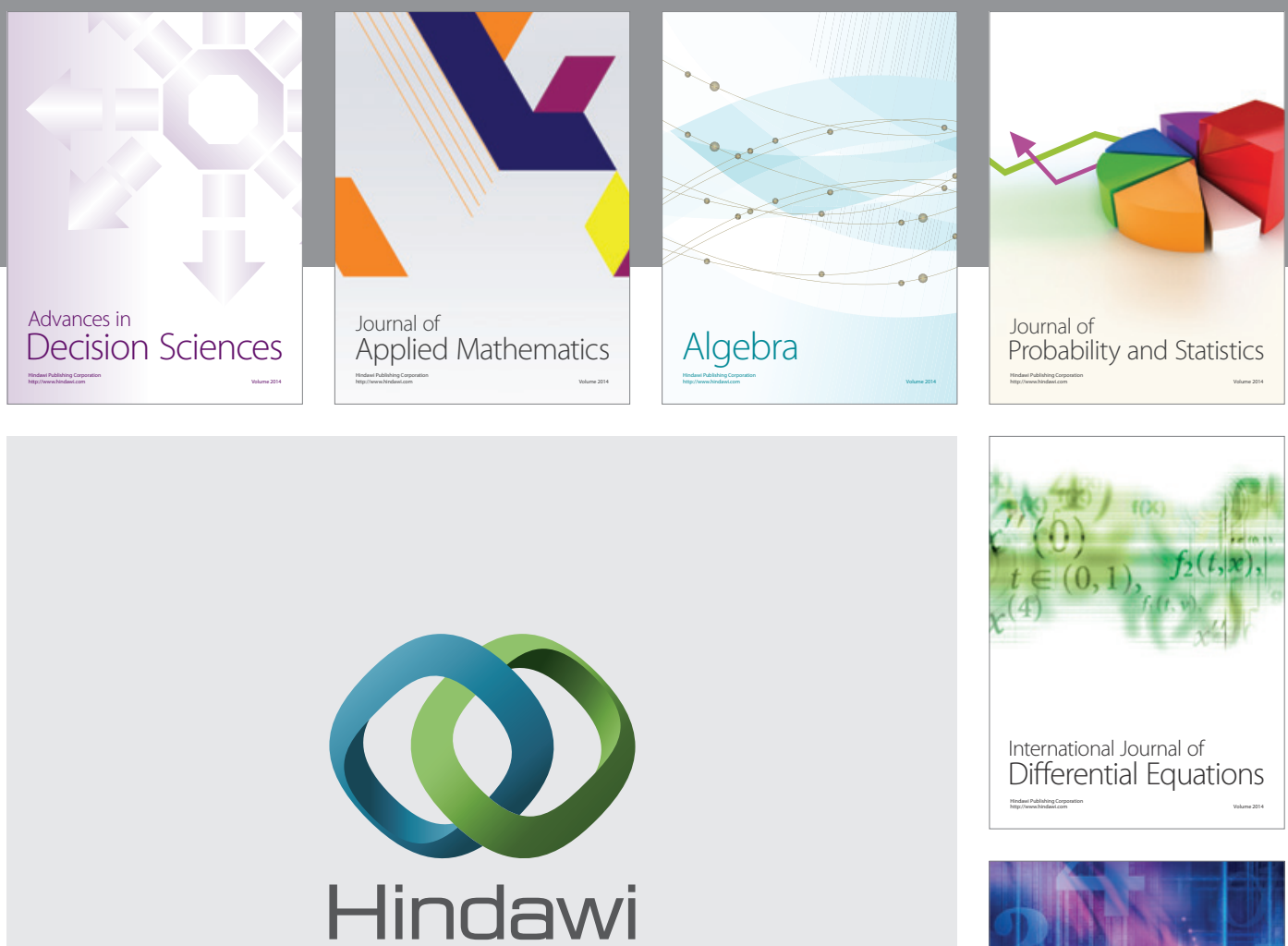

Submit your manuscripts at http://www.hindawi.com
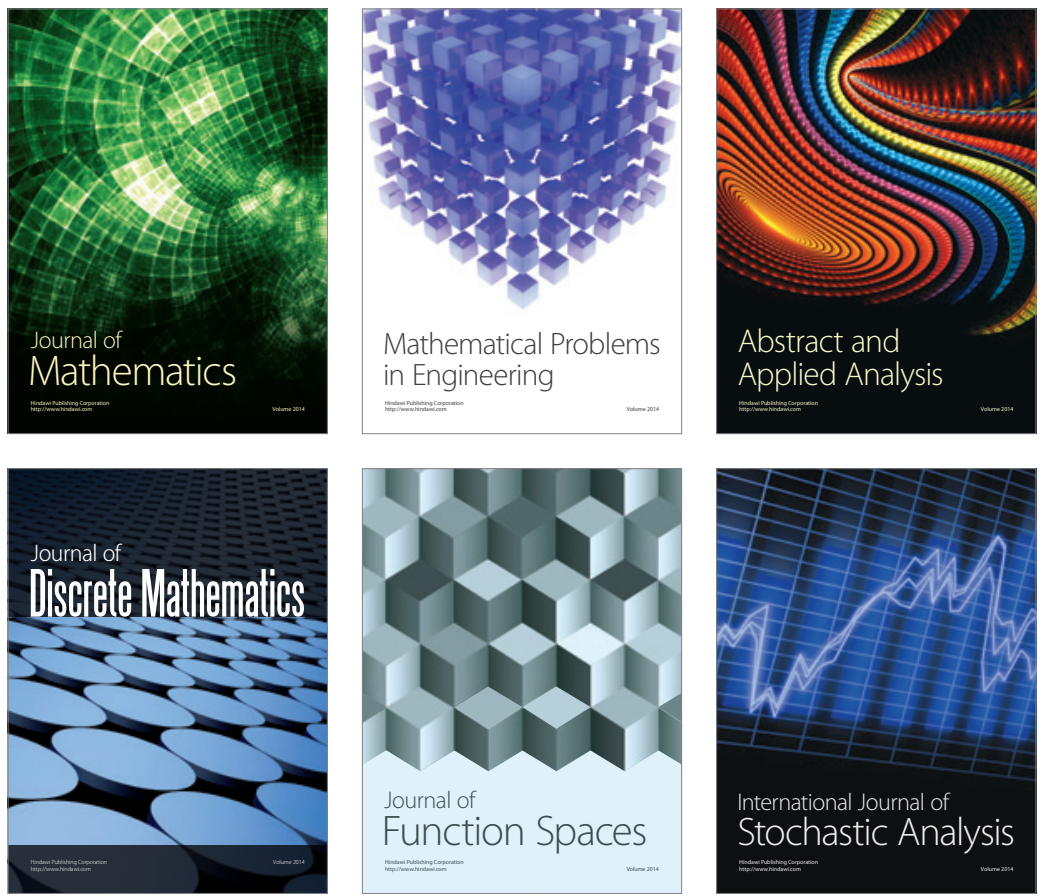

Journal of

Function Spaces

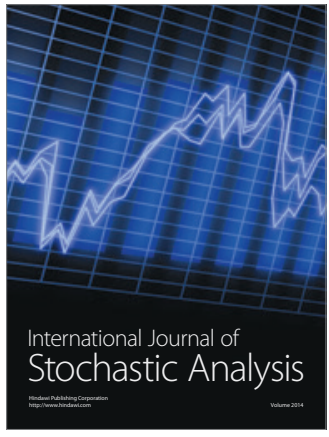

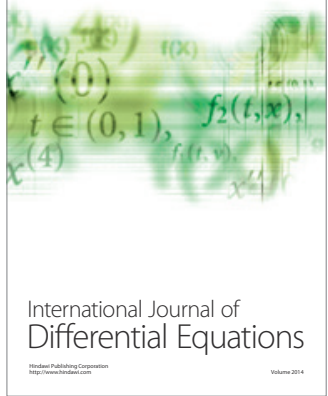
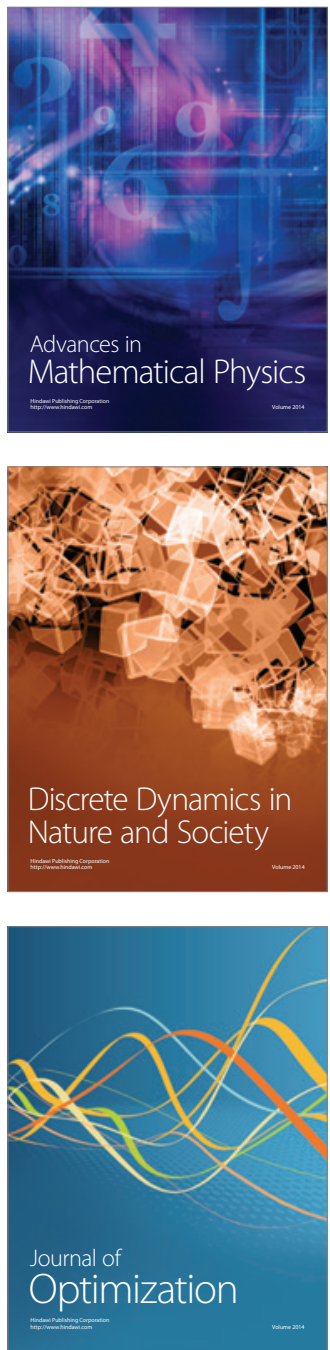